\title{
Mutant number distribution in an exponentially growing population
}

\author{
Peter Keller and Tibor Antal \\ School of Mathematics, University of Edinburgh, Peter Guthrie Tait Road, \\ Edinburgh EH9 3FD, UK \\ E-mail: peter.keller@ed.ac.uk
}

Received 14 October 2014

Accepted for publication 26 November 2014

Published 9 January 2015

Online at stacks.iop.org/JSTAT/2015/P01011

doi:10.1088/1742-5468/2015/01/P01011

\begin{abstract}
We present an explicit solution to a classic model of cell-population growth introduced by Luria and Delbrück (1943 Genetics 28 491-511) 70 years ago to study the emergence of mutations in bacterial populations. In this model a wild-type population is assumed to grow exponentially in a deterministic fashion. Proportional to the wild-type population size, mutants arrive randomly and initiate new sub-populations of mutants that grow stochastically according to a supercritical birth and death process. We give an exact expression for the generating function of the total number of mutants at a given wild-type population size. We present a simple expression for the probability of finding no mutants, and a recursion formula for the probability of finding a given number of mutants. In the 'large population-small mutation' limit we recover recent results of Kessler and Levine (2014 J. Stat. Phys. doi:10.1007/s10955-014-1143-3) for a fully stochastic version of the process.
\end{abstract}

Keywords: stochastic processes (theory), mutational and evolutionary processes (theory), population dynamics (theory)

ArXiv ePrint: 1410.3307 


\section{Contents}

1. Introduction 2

2. General population size functions 4

3. Generating function for exponential growth 5

4. Special case of neutral mutations, $\gamma=1 \quad 7$

5. Limit behavior $\quad 8$

5.1. Large population-small mutation limit $\ldots \ldots \ldots \ldots \ldots \ldots \ldots \ldots \ldots \ldots$

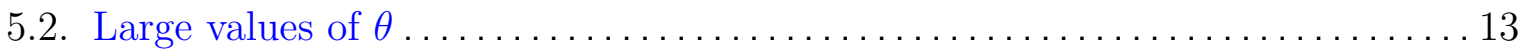

5.3. Large population limit at fixed mutation rate $\ldots \ldots \ldots \ldots \ldots \ldots \ldots \ldots \ldots \ldots$

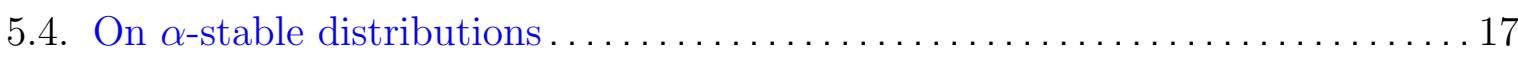

$\begin{array}{ll}\text { 6. Tail behavior } & 18\end{array}$

7. Discussion and summary 21

Acknowledgments $\quad 22$

Appendix A. Proof of recursion formulas 22

Appendix B. Large $\gamma$-asymptotics of the resistance probability 24

Appendix C. Definitions and useful identities 25

$\begin{array}{ll}\text { Appendix D. Theorems from singularity analysis } & 27\end{array}$

$\begin{array}{ll}\text { References } & 28\end{array}$

\section{Introduction}

When a population of bacteria is attacked by a lethal virus, often a sub-population survives. At the beginning of the 1940's an important question was whether this resistance is due to adaptation which is induced under the stress of attack, or is simply due to mutations that occurred beforehand during the expansion of the population. To clarify this question, Luria and Delbrück conducted their now famous experiments in 1943 [1], and showed that indeed the natural variability of cells can withhold a sub-population from extinction. They formulated a simple mathematical model in which both wild-type and the mutant cells grow deterministically, but the mutants appear randomly, proportional to the wild-type population size. They derived many properties of the model, in particular for the mutant size distribution and proposed a method to estimate the mutation rate from data. 
In the seminal paper of Lea and Coulson [3], the original model was extended to allow stochastic growth of the mutant population as a pure birth process. They derived the distribution of the number of mutants for the first time for neutral mutation. In 1955 Bailey published elegant computations and some results on his own modifications of the process, see [4]. Since then many efforts have been undertaken to understand the process better. The review paper of Zheng [5], gives a formidable overview of the history of the process and clarifies most concerns related to the infinite moments of the proposed distributions.

New interest has kindled recently in the mutant distribution of the fully stochastic version, where wild-type cells grows according to a birth and death process. Including cell death into the model extended the range of its possible applications. This model was formulated by Kendall [6], and a full solution was provided in [7], where the Kolmogorov equations for the generating function of both cell types were solved explicitly. From the generating function the joint probability of a given number of wild type and mutant cells can be obtained for any finite times. Expressions for finite times become important for experimental studies [8-10], where the asymptotic limit might be out of reach.

In many situations, most notably in the study of mutations in tumor growth $[11,12]$, the age of the wild-type population is rarely known. At tumor detection we have a fairly good idea about the size of the tumor but the time of its initiation is unknown. This led to studies of the mutant distribution at a fixed size of wild-type population. For neutral mutataion and pure birth processes this problem was solved by Angerer [13]. Iwasa, Nowak and Michor [14] extended this model to non-neutral mutants and to birth-anddeath processes. They derive mutant distributions and resistance probability assuming the product of population size and mutation rate to be small. Komarova suggested a very elegant method to obtain an approximate mutant distribution [15]. More recently, in two remarkable papers $[2,16]$ Kessler and Levine obtained the full mutant distribution for a large but fixed size wild-type population. They used approximate methods to simplify the Kolmogorov equations, and in an independent derivation they also used the exact solution of the fully stochastic case given in [7]. By letting the previously constant product of mutation rate and population size go to infinity, they derive $\alpha$-stable distributions. For the same limit, similar results were derived with other methods by Durrett and Moseley [17] for beneficial mutations. This result was already given, but not proven, by Mandelbrot in [18]. Moehle treats the classic case of neutral mutation utilizing Compound Poisson processes in [19]. In [20] Janson treats a similar model with fixed, non-random number of offspring, by mapping a reducible multi-type branching process to Pólya-Urns and investigates several limits.

In this paper we make the assumption that the wild-type population grows according to a deterministic exponential function. This is valid in many biological contexts, in particular when the members of a population do not compete for resources. An example are early stages of cancer, where the tumor-cell population is large, but not yet effected significantly by resource constrains. In treatment with chemotherapy, the rise of resistant sub-populations is of paramount importance, since resistant cells (mutants) are the main cause for a treatment failure. Moreover, the introduction of non-exponential tumor growth is not only difficult to treat mathematically, but is also a highly disputed topic in oncology. A detailed discussion and comparison with medical studies of exponential and 
non-exponential growth models can be found in [21]. For the sake of simplicity we shall treat exponential growth only.

We introduce and review the general ansatz of Dewanji [22] for arbitrary growth functions in section 2 and obtain the generating function of the number of mutants explicitly in terms of hypergeometric functions for the exponential case in section 3 . The special case of neutral mutations is discussed separately in section 4 . We investigate various limits of the mutant distribution in section 5. We recover all corresponding results from the above mentioned papers for general parameters and extend them to finite wildtype populations, to mutants with explicit death, and to deleterious (disadvantageous compared to wild-type) mutations. In addition to analyzing the mean and the variance of the number of mutants, we characterize the shape of the mutant number distribution, numerically study its mode, give the probability of resistance, and the distribution of the sizes of individual clones. Relations to Compound Poisson processes, the Yule-Simon distribution, and $\alpha$-stable distributions are also discussed. We give a recursion to calculate the probability distribution of the mutants efficiently and analyze the distribution's tail behavior in-depth in the final section, thereby extending the results of [23, 24]. In the Summary we show that for small mutation rates the mutant distribution obtained for the semi-deterministic model agrees very well with numerical results obtained for the fully stochastic version of the model.

\section{General population size functions}

We consider a cell population that consists of two types of cells, a wild type (type A) and a mutant (type B). Each $A$-cell independently of all other cells produces a mutant $B$-cell at rate $\nu$. If we approximate the size of the $A$-cell population via the deterministic function $f(t)$, so that mutants are produced at rate $\nu f(t)$, the arrival times of new mutants follow a non-homogeneous Poisson process. Each $B$-cell descended from an $A$-cell at time $s<t$ is the initiator of a new sub-population of mutants (a clone), whose size we denote by $Y_{k}$. At time $t$ the total number $K$ of clones is a Poisson random variable with mean

$$
m=\mathbb{E}(K)=\int_{0}^{t} \nu f(s) \mathrm{d} s .
$$

We assume that clones develop independently as some stochastic process with generating function $g_{t}(z)=\mathbb{E}\left(z^{Y}\right)$. Since each clone $Y_{k}$ is generated according to a Poisson process, the family $\left(Y_{i}\right)_{i \in\{1, \ldots, K\}}$ is independent, identically distributed (iid) and the generating function of each clone is

$$
\psi(z)=\mathbb{E}\left(z^{Y}\right)=\frac{\nu}{m} \int_{0}^{t} f(s) g_{t-s}(z) \mathrm{d} s .
$$

The total number $B_{t}$ of mutants at time $t$ is a Compound Poisson random variable

$$
B_{t}=\sum_{i=1}^{K} Y_{i}
$$

Using conditional expectation, the generating function of $B_{t}$ can be written as

$$
G(z)=\mathbb{E}\left(z^{B_{t}}\right)=\mathbb{E}\left(\mathbb{E}\left(z^{B_{t}} \mid K\right)\right) .
$$

Now

$$
\mathbb{E}\left(z^{B_{t}} \mid K=k\right)=\psi^{k}(z)
$$

doi:10.1088/1742-5468/2015/01/P01011 
since the clones are independent and thus

$$
G(z)=\mathbb{E}\left(\psi^{K}\right)=\sum_{k \geqslant 0} \frac{(\psi(z) m)^{k}}{k !} \mathrm{e}^{-m}=\mathrm{e}^{m(\psi(z)-1)},
$$

which appears in [19] and is characteristic for Compound Poisson variables. Using (1) and (2), we can also write

$$
G(z)=\exp \left(\nu \int_{0}^{t} f(s)\left[g_{t-s}(z)-1\right] \mathrm{d} s\right)
$$

which appears in [22] in a more general setting.

Since the generating function of $B_{t}$ is of exponential form, we introduce the following notation for arbitrary random variable $X$

$$
\Lambda_{X}(z)=\log \mathbb{E}\left(z^{X}\right)=\log G_{X}(z)
$$

and refer to $\Lambda_{X}(z)$ as the log-generating function of $X$.

\section{Generating function for exponential growth}

Let us consider the special case of an exponentially growing wild-type population, such that $f(t)=\mathrm{e}^{\delta t}$, for some $\delta>0$. Hence mutants are produced at rate $\nu \mathrm{e}^{\delta t}$. Moreover, let us assume that each clone behaves like a linear birth-death process with birth rate $\alpha$ and death rate $\beta$ with positive relative fitness $\lambda=\alpha-\beta>0$, i.e. the process is supercritical. The extinction probability of a mutant clone is $q=\beta / \alpha=1-\lambda / \alpha$, and its generating function is also well known [25]

$$
g_{s}(z)=1-\frac{1-q}{1-\xi \mathrm{e}^{-\lambda s}}, \quad \xi=\frac{q-z}{1-z}=1-\frac{1-q}{1-z} .
$$

The pure birth case of $\beta=0$ and thus $q=0$ is well studied and corresponds to the assumption that cells only divide, but never die.

We are interested in the distribution of the number of mutants at the time when the number of $A$-cells reaches exactly $N$. Since the $A$-cells grow deterministically, this happens at time $\tau=\log (N) / \delta$. We use the shorthand notation $B \equiv B_{\tau}$ for the number of mutants at time $\tau$. Therefore the mutant log-generating function (4) becomes

$$
\Lambda_{B}(z)=\nu \int_{0}^{\tau} \mathrm{e}^{\delta s}\left(g_{\tau-s}(z)-1\right) \mathrm{d} s=\frac{\mu}{\gamma} \int_{0}^{\tau} \frac{1}{\mathrm{e}^{\lambda s} N^{-1 / \gamma} \xi-1} \delta \mathrm{e}^{\delta s} \mathrm{~d} s
$$

where

$$
\gamma=\delta / \lambda \text { and } \mu=\nu / \alpha
$$

After a change of variable $u=\mathrm{e}^{\delta s} / N$ we can compute the integral

$$
\begin{aligned}
\Lambda_{B}(z) & =\frac{-N \mu}{\gamma} \int_{1 / N}^{1} \frac{1}{1-u^{1 / \gamma} \xi} \mathrm{d} u \\
& =\frac{-N \mu}{\gamma} \int_{1 / N}^{1} \sum_{k \geqslant 0}\left(u^{1 / \gamma} \xi\right)^{k} \mathrm{~d} u=-\left.N \mu \sum_{k \geqslant 0} \xi^{k} \frac{u^{(k+1) / \gamma}}{\gamma+k}\right|_{1 / N} ^{1} \\
& =\mu \sum_{k \geqslant 0} \xi^{k}\left[\frac{N^{-k / \gamma}}{\gamma+k}-\frac{N}{\gamma+k}\right] .
\end{aligned}
$$




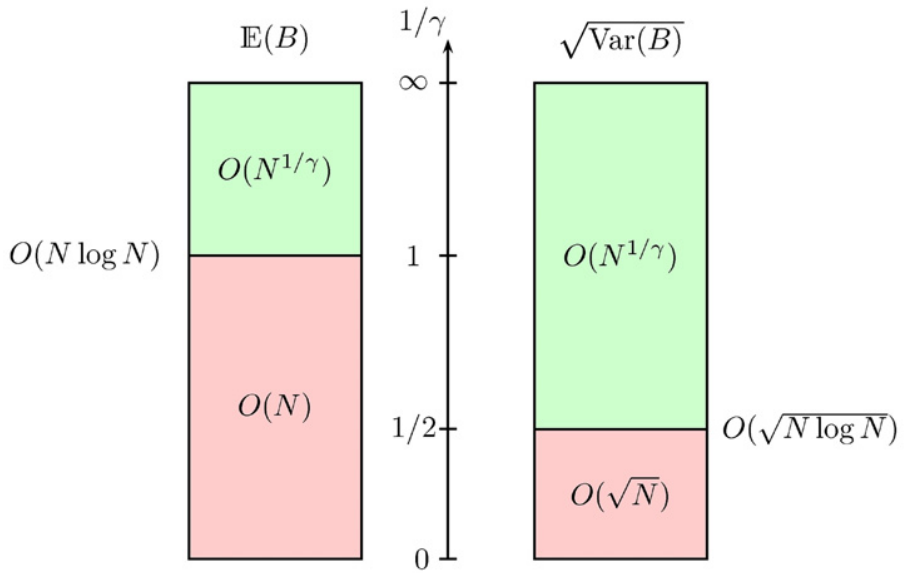

Figure 1. Orders of the mean number of mutants $\mathbb{E}(B)$ and its standard deviation $\sqrt{\operatorname{Var}(B)}$ for large $N$. Mutants have a fitness advantage for $1 / \gamma>1$ and a disadvantage for $1 / \gamma<1$ with respect to the wild-type cells.

We can rewrite (6) in terms of the hypergeometric function (C.6)

$$
\Lambda_{B}(z)=\log G_{B}(z)=\frac{N \mu}{\gamma}\left[\frac{1}{N} F\left(\begin{array}{c}
1, \gamma \\
1+\gamma
\end{array} ; \xi N^{-1 / \gamma}\right)-F\left(\begin{array}{c}
1, \gamma \\
1+\gamma
\end{array} ; \xi\right)\right],
$$

where we utilized the Pochhammer symbol $(\gamma)_{k}$ (C.5) to verify that

$$
\frac{\gamma}{\gamma+k}=\frac{(\gamma)_{k}}{(1+\gamma)_{k}}
$$

The above equation (7) is the final, exact, closed-form solution for the mutant distribution for an exponentially growing wild type population. In the rest of the paper we shall analyze its properties.

The mean and variance of the number of mutants can be calculated by taking the usual approach of differentiating the generating function (7) or by using Dewanji's general expressions for mean and variance for the case of arbitrary growth function $f(t)$, see [22]; this results in

$$
\mathbb{E}(B)=\frac{N \mu}{1-q} \cdot \begin{cases}\log N & \gamma=1 \\ \frac{1}{1-\gamma}\left(N^{1 / \gamma-1}-1\right) . & \gamma \neq 1\end{cases}
$$

and

$$
\operatorname{Var}(B)=\frac{N \mu}{(1-q)^{2}} \cdot \begin{cases}2(N-1)-(1+q) \log N & \gamma=1 \\ (1+q)\left(N^{-1 / 2}-1\right)+\log N & \gamma=2 \\ \frac{2}{2-\gamma} N^{2 / \gamma-1}+\frac{1+q}{\gamma-1} N^{1 / \gamma-1}+\frac{q(2-\gamma)+\gamma}{(2-\gamma)(1-\gamma)} & \gamma \notin\{1,2\}\end{cases}
$$

These expressions generalize those given in Zheng [5] (replace $N=\exp (\delta t)$ and $\delta \equiv \beta_{1}$, $\lambda \equiv \beta_{2}$ in $[5,(52)$ and (53)]).

We give an overview of the large $N$ behavior of the expectation and the variance of the number of mutants $B$ in figure 1 . The mean number of deleterious mutants $(1 / \gamma<1)$ is of the same order as the wild type cells. However, the number of advantageous mutants $(1 / \gamma>1)$ is growing faster than the wild type population. Note also that for advantageous 
mutants $(1 / \gamma>1)$ the mean and the standard deviation have the same order, which implies that the fluctuations are important and a stochastic description of the process is essential. Only for very deleterious mutants $(1 / \gamma<1 / 2)$ the process becomes selfaveraging, and the fluctuations become as predicted by the central limit theorem. For intermediate deleterious mutants $(1 / 2<1 / \gamma<1)$ the relative standard deviation varies continuously with $\gamma$.

We can obtain the probabilities $p_{k}=\mathbb{P}(B=k)$ by Taylor expanding $G(z)$ in $z$, or by using the Gauss inversion formula. Since this is computationally intense, we give a recursive formula for the probabilities instead

$$
p_{n}= \begin{cases}\mathrm{e}^{q_{0}}, & n=0 \\ \frac{1}{n} \sum_{k=0}^{n-1}(n-k) q_{n-k} p_{k}, & n \geqslant 1,\end{cases}
$$

where

$$
q_{0}=\frac{N \mu}{\gamma}\left[\frac{1}{N} F\left(\begin{array}{c}
1, \gamma \\
1+\gamma
\end{array} ; N^{-1 / \gamma} q\right)-F\left(\begin{array}{c}
1, \gamma \\
1+\gamma
\end{array} ; q\right)\right]
$$

and for $k \geqslant 1$

$$
\begin{gathered}
q_{k}=\mu \sum_{j=1}^{k}\left(\begin{array}{c}
k-1 \\
j-1
\end{array}\right) \frac{1}{j+\gamma}\left(\frac{1-q}{q-N^{1 / \gamma}}\right)^{j} F\left(\begin{array}{c}
1, \gamma \\
1+\gamma+j
\end{array} ; N^{-1 / \gamma} q\right) \\
+N \mu \frac{(k-1) !}{(\gamma+1)_{k}} F\left(\begin{array}{c}
k, \gamma \\
1+\gamma+k
\end{array} ; q\right) .
\end{gathered}
$$

We give a proof of this recursion in appendix A.

\section{Special case of neutral mutations, $\gamma=1$}

Often there is interest in mutations which do not change the behavior of the cell, so called neutral mutations. In this special case when $\gamma=1$, that is $\delta=\lambda$, we can further simplify the log-generating function $\Lambda_{B}(z)$ given in (7), by using (C.17). Alternatively, by using the series expansion

$$
\log (1-z)=-\sum_{k \geqslant 1} \frac{z^{k}}{k}
$$

we can rewrite (6) for $\gamma=1$ as

$$
\Lambda_{B}(z)=\mu \sum_{k \geqslant 0} \xi^{k}\left[\frac{N^{-k}}{k+1}-\frac{N}{k+1}\right]=\frac{N \mu}{\xi} \log \left(\frac{1-\xi}{1-\xi / N}\right) .
$$

Hence the generating function becomes

$$
G(z)=\left(\frac{1-\xi}{1-\xi / N}\right)^{\frac{N \mu}{\xi}}
$$

By introducing the variables

$$
y=\frac{z-q}{1-q}=\frac{\xi}{\xi-1}, \quad \phi=1-\frac{1}{N}, \quad \theta=N \mu,
$$

doi:10.1088/1742-5468/2015/01/P01011 
we obtain the form

$$
G(z)=(1-\phi y)^{\theta \frac{1-y}{y}} .
$$

If we further specialize to mutant cells that cannot die, that is $\beta=0$ (which implies $q=0$ and $y=z$ ), we find

$$
G(z)=(1-\phi z)^{\theta \frac{1-z}{z}} .
$$

This formula was first derived in [3], and also given in [5] with some historical perspective.

The coefficients in the recursion formula (10) become simpler for $\gamma=1$

$$
q_{k}= \begin{cases}-\frac{\theta}{q} \log \left(1+\phi \frac{q}{1-q}\right) & k=0 \\
\frac{\theta \phi^{k}}{[1-q(1-\phi)]^{k}}\left[\frac{1}{k}-\frac{\phi}{k+1} F\left(\begin{array}{c}
1,1 \\
2+k
\end{array} ;-\frac{\phi q}{1-q}\right)\right] & k \geqslant 1\end{cases}
$$

which is also derived in appendix A. Note that

$$
p_{0}=P(B=0)=\mathrm{e}^{q_{0}}=\left(1+\frac{q \phi}{1-q}\right)^{-\theta / q}
$$

and all other $p_{n}$ probabilities can be obtained from recursion (10). When mutants do not die, i.e. $\beta=0$ (which implies $y=z$ ), the coefficients further simplify to

$$
q_{k}= \begin{cases}-\theta \phi & k=0 \\ \theta\left(\frac{\phi^{k}}{k}-\frac{\phi^{k+1}}{k+1}\right) & k \geqslant 0,\end{cases}
$$

as given in [5, p 18]. Note that the coefficients $q_{k}$ appear in [14, appendix D] as the approximated probabilities for large $N$ and $N \mu \ll 1$, in which case $\phi \approx 1$.

In the $N \rightarrow \infty$ limit with $\theta$ kept constant, we have $\phi=1$ and the above recursion simplifies to

$$
p_{0}=\mathrm{e}^{-\theta}, \quad n p_{n}=\theta \sum_{i=0}^{n-1} \frac{p_{i}}{n-i+1},
$$

which was derived in $[26,27]$. Note that (17) tends to the generating function of the Luria-Delbrück distribution in this case. We will consider this limit in more detail and for general $q$ and $\gamma$ in the next section.

\section{Limit behavior}

In section 3 we derived a recursion for the exact probability distribution of the number of mutants present at time $\tau=\frac{\log N}{\delta}$, under the assumption of exponential wild-typegrowth. The coefficients of this recursion, given in (12), are quite complicated, so we seek for an easier limit case. In (7), the first addend stems from the lower integral boundary 0 and should vanish for large $\tau$ resp. large $N$ and small mutation rate. This turns out to be true only if $\theta=N \mu$ is held constant. The resulting distribution is a Compound Poisson random variable, enabling us to directly determine the distribution of the size of a clone, i.e. the sub-population size of mutants descended from one original mutant. This distribution shows already a power-law tail, which will be discussed in section 6 . 


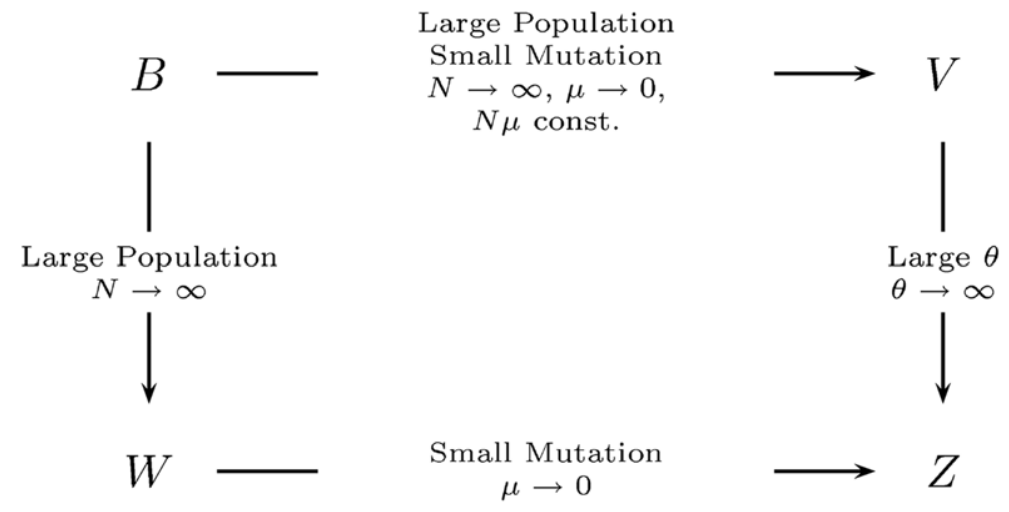

Figure 2. A schematic overview of the possible limits. All limits are convergencein-distribution results.

In applications, $\theta$ can be large, hence we discuss a consecutive limit in which $\theta$ goes to infinity. Since a Compound Poisson random variable is the sum of a random number of i.i.d. variables, it is not surprising that the distribution of the limit variable, which we call $Z$, is $\alpha$-stable. Although the theory of generalized limit theorems is rich, see for example [28], we use our explicit results for the generating function to perform the limit directly. Since we consider only the convergence of generating functions or Laplacetransforms, all limits in this paper are meant as convergence in distribution.

Note that in [17], a non-rigorous proof was given for the direct limit from $B$ to $Z$ (see figure 2) for the fully stochastic case in a slightly different setting for $0<\gamma<1$. It utilizes an approximation of the wild-type population size by a deterministic exponential growth function, that stems from the fact that in the two-type, fully stochastic model the wildtype population behaves like a one-type birth and death process if the mutation rate $\nu$ is very small. Then the number of $A$-cells can be approximated by $\exp (\delta t) X, X \sim \operatorname{Exp}(1)$. A similar limit in [19] covers the $\gamma=1$ case of the classic Luria Delbrück distribution.

Another limit approach is to fix the mutation rate and let $N \rightarrow \infty$ under a proper rescaling. Results for this limit were presented in [18] and their derivation given in a privately distributed second part of the paper, which is no longer available. We reproduce these results in terms of hypergeometric functions and then take the limit $\mu \rightarrow 0$ under a similar scaling as in the $N \mu$-constant case, to recover once again the limit variable $Z$.

\subsection{Large population-small mutation limit}

In applications, the mutation rate $\nu$ is usually small, while the population size $N$ is very large. We therefore investigate the simultaneous limit $N \rightarrow \infty$ and $\mu=\nu / \alpha \rightarrow 0$, such that $\theta=N \mu$ is held constant. We call this limit Large Population-Small Mutation Limit (LPSM). Note that we introduced $\theta$ already in (15), in analogy to the notation introduced in $[5]$.

Formally we can express the LPSM-limit as

$$
\lim _{\substack{N \rightarrow \infty, \mu \rightarrow 0 \\ \theta \text { const. }}} B=V
$$

where $V$ is the limiting random variable, which we characterize via its log-generating function. When $\theta$ is held constant, the log-generating function of $B$, given in (7), depends 
Table 1. Overview of mean and variance of $V$, depending on $\gamma$.

\begin{tabular}{lll}
\hline & $\mathbb{E}(V)$ & $\operatorname{Var}(V)$ \\
\hline$\gamma>2$ & $\frac{\theta}{(1-q)(\gamma-1)}$ & $\frac{\theta}{(1-q)^{2}}\left(\frac{q(2-\gamma)+\gamma}{(\gamma-2)(\gamma-1)}\right)$ \\
$1<\gamma \leqslant 2$ & $\frac{\theta}{(1-q)(\gamma-1)}$ & $\infty$ \\
$0<\gamma \leqslant 1$ & $\infty$ & $\infty$ \\
\hline
\end{tabular}

only in the first addend on $N$. We therefore expand the first addend into a power series in $\xi$. For arbitrary $\gamma>0$

$$
\frac{1}{N} F\left(\begin{array}{c}
1, \gamma \\
1+\gamma
\end{array} ; N^{-1 / \gamma} \xi\right)=\sum_{k \geqslant 0} \frac{\gamma}{\gamma+k} \xi^{k} \frac{1}{N^{k / \gamma+1}} \rightarrow 0, \text { as } N \rightarrow \infty .
$$

Thus the immediate result for the LPSM-limit is

$$
\Lambda_{V} z=\lim _{\substack{N \rightarrow \infty, \mu \rightarrow 0 \\
\theta \text { const. }}} \Lambda_{B}(z)=-\frac{\theta}{\gamma} F\left(\begin{array}{c}
1, \gamma \\
1+\gamma
\end{array} ; \xi\right) .
$$

which we can rewrite in terms of $y=\frac{z-q}{1-q}$ by using (C.10)

$$
\Lambda_{V}(z)=\frac{\theta}{\gamma}(1-y) F\left(\begin{array}{c}
1,1 \\
1+\gamma
\end{array} ; y\right)
$$

This expression is the second addend in the general formula (7), so we can adapt the recursion (10) for the probability distribution of $V$ easily. This yields the recursion coefficients

$$
q_{k}= \begin{cases}-\frac{\theta}{\gamma} F\left(\begin{array}{c}
1, \gamma \\
1+\gamma
\end{array} ; q\right), & k=0 \\
\theta \frac{(k-1) !}{(\gamma+1)_{k}} F\left(\begin{array}{c}
k, \gamma \\
1+\gamma+k
\end{array} ; q\right), & k \geqslant 1\end{cases}
$$

Note that the coefficients $q_{k}$ appear in $[14,(16)]$ as the approximated probabilities for large $N$ and $N \mu \ll 1$. For the special case of $q=0$ and $\gamma=1$ the distribution of $V$ is the famous Luria-Delbrück distribution, for which Ma gave a simple recursion in [26].

The expectation and variance of $V$ can be derived as usual via derivatives of the generating function. The computations are tedious, in particular because the convergence behavior of the hypergeometric function depends on $\gamma$, but not very interesting. We give the results in table 1 . Note that they are consistent with the application of the $N \rightarrow \infty$, $\mu \rightarrow 0$ limit directly to the mean and variance of $B$ given in (8) and (9). Interestingly, the mean is finite only for $\gamma>1$ and the variance only for $\gamma>2$.

We note that the LPSM-limit is independent of the initial number $N_{0}$ of wild-type cells. This can be easily seen, when we choose $f(t)=N_{0} \mathrm{e}^{\delta t}$ in (4). Then the integral representation of the log-generating function (5) is just $G_{B}^{N_{0}}(z)$. This property directly mimics the branching property of a fully stochastic two-type branching process. Adapting the calculations from (6), the log-generating function

$\Lambda_{B}(z)=\log G_{B}^{N_{0}}(z)=\frac{N \mu}{\gamma}\left[\frac{N_{0}}{N} F\left(\begin{array}{c}1, \gamma \\ 1+\gamma\end{array} ; \xi \frac{N^{-1 / \gamma}}{N_{0}}\right)-F\left(\begin{array}{c}1, \gamma \\ 1+\gamma\end{array} ; \xi\right)\right]$. 
Again, in the LPSM-limit only the first addend depends on $N$ resp. $N_{0}$ and vanishes with $N \rightarrow \infty$.

Note further, that the limit log-generating function $\Lambda_{V}(z)$ has a direct interpretation with respect to our initial model. We write

$$
\Lambda_{V}(z)=-\frac{\theta}{\gamma} F\left(\begin{array}{c}
1, \gamma \\
1+\gamma
\end{array} ; \xi\right)=\frac{\theta}{\gamma} \int_{0}^{1} \frac{1}{u^{1 / \gamma} \xi-1} \mathrm{~d} u
$$

by (C.8). A change of variables with $s=\frac{\log (u N)}{\delta}$ gives

$$
\frac{\theta}{\gamma} \int_{0}^{1} \frac{1}{u^{1 / \gamma} \xi-1} \mathrm{~d} u=\frac{\theta}{\gamma N} \int_{-\infty}^{\tau} \frac{1}{\mathrm{e}^{\lambda s} N^{-1 / \gamma} \xi-1} \delta \mathrm{e}^{\delta s} \mathrm{~d} s .
$$

Indeed, $\Lambda_{V}(z)$ is the generating function of the model started at $-\infty$ instead of zero.

By another change of variables $t=\tau-s$

$$
\Lambda_{V}(z)=\frac{\theta}{\gamma} \int_{0}^{\infty} \frac{1}{\mathrm{e}^{-\lambda t} \xi-1} \delta \mathrm{e}^{-\delta t} \mathrm{~d} t=\frac{\theta}{(1-q) \gamma} \int_{0}^{\infty}\left(g_{t}(z)-1\right) \delta \mathrm{e}^{-\delta t} \mathrm{~d} t .
$$

Let $X \sim \operatorname{Exp}(\delta)$ be an exponential random variable with mean $1 / \delta$, then

$$
\Lambda_{V}(z)=\frac{\theta}{(1-q) \gamma} \mathbb{E}\left[g_{X}(z)-1\right] .
$$

This is the log-generating function of a Compound Poisson random variable, where

$$
\mathbb{E}\left[g_{X}(z)\right]=1-(1-q) F\left(\begin{array}{c}
1, \gamma \\
1+\gamma
\end{array} ; \xi\right)
$$

Via the usual interpretation of a Compound Poisson, the limiting number of clones (i.e. the number of actual mutation events) has a Poisson distribution with intensity $\frac{\theta}{(1-q) \gamma}$ and $\mathbb{E}\left[\phi_{X}(z)\right]$ describes the clone size distribution, that is the size of the sub-population founded by a single original mutant. For $q=0$ the generating function (25) is the generating function of a Yule-Simon distribution with parameter $\gamma$ and is generated by a geometric distribution $\operatorname{Geo}(p)$ with random parameter $p=\exp (-Y), Y \sim \operatorname{Exp}(\gamma)$. This distribution is already a heavy-tailed distribution with infinite mean for $0<\gamma \leqslant 1$.

We identified the distribution of $V$ as (discrete) Compound Poisson random variable and argued that it has infinite mean for $0 \leqslant \gamma \leqslant 1$ and infinite variance for $0 \leqslant \gamma \leqslant 2$, see table 1 . Since the potentially non-finiteness of the moments makes the application of moment-methods very difficult and unreliable, we give some information about the mode of $V$. The mode is defined as the value $\mathrm{k}$ at which the probability mass function of $V$ takes its maximum. In uni-modal distributions the mode corresponds to the peak of the distribution.

From numerical analysis we are confident that the distribution of $V$ is indeed unimodal, as can be seen in figure 3 where we vary the parameters $\gamma$ and $\theta$. In general, however, it is difficult to derive an explicit formula since we only have the recursion (23) available. The recursion, however, makes the first few probabilities explicitly accessible. We therefore investigate the ratio

$$
\frac{p_{1}}{p_{0}}=\frac{\theta}{\gamma+1} F\left(\begin{array}{c}
1, \gamma \\
2+\gamma
\end{array} ; q\right)
$$

This ratio is less than one, if the probability $p_{0}$ is the maximum of the distribution and larger if the maximum is bigger or equal to one. This ratio depends not only on $\theta$ and 
Mutant number distribution in an exponentially growing population
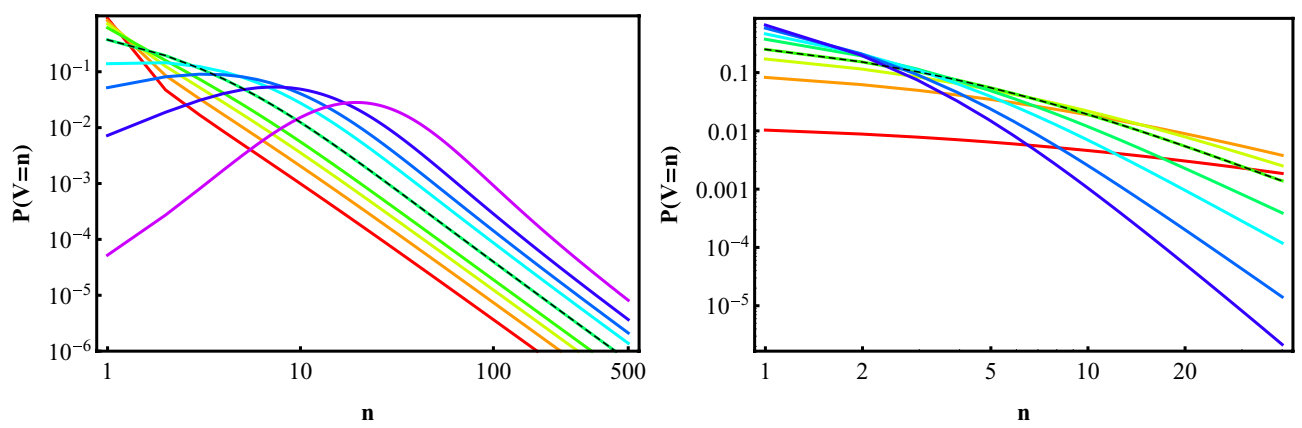

Figure 3. On the left, we plot the probability distribution of the number of mutants in the large population-small mutation limit with $\theta=N \mu$ constant. While keeping $\gamma=1.5$ and $q=1 / 2$ constant, we vary $\theta=1 / 10,1 / 5,1 / 3,1 / 2,1$, $2,3,5,10$, beginning with the lowest (red) line. On the right, we vary $\gamma$ choosing $0.25,0.5,0.75,1,1.5,2,3$ and 4 , beginning with the horizontal (red) line, for $\theta=1$ and $q=1 / 2$. The case $\gamma=1$ is indicated with a dashed line in both figures. We used recursion (23) to calculate the probabilities.

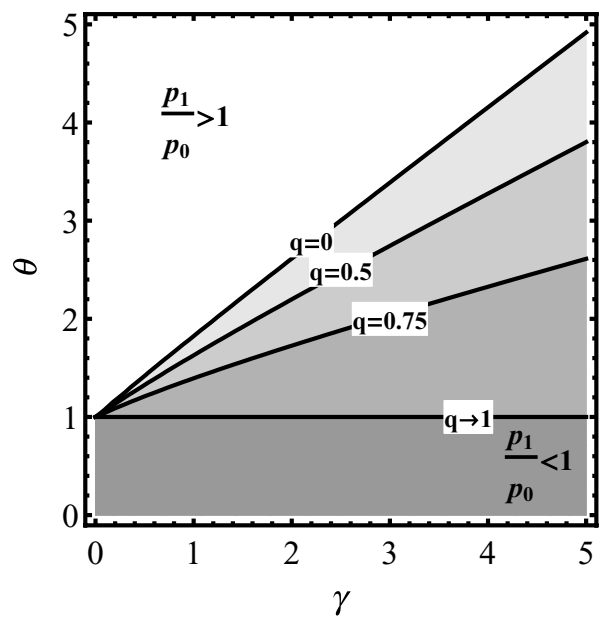

Figure 4. Comparison of the probability of no mutants $p_{0}$ and a single mutant $p_{1}$. Solid lines indicate $p_{0}=p_{1}$ depending on $q$. We included the extreme case $q=1$ as well.

$\gamma$, but also on $q$. In figure 4 we show a phase diagram on the $\theta-\gamma$ plane displaying the boundary of regions where no mutants are the most probable, for a few values of $q$. The plot shows that the boundaries behave like a linear function. This is can be confirmed, if we set $p_{1} / p_{0}=1$ in (26), then $\theta$ and $\gamma$ can be seperated and the boundary is described by

$$
\theta=(1+\gamma) F\left(\begin{array}{c}
1, \gamma \\
2+\gamma
\end{array} ; q\right)^{-1} \sim 1+q+(1-q) \gamma+O\left(\gamma^{2}\right), \text { for large } \gamma
$$

The last approximation is due to an asymptotic result for the hypergeometric function, which we derive in appendix $\mathrm{B}$. The ratio $p_{1} / p_{0}$, however, gives no information about the actual value of the mode(B), that is the most probable number of mutants. By numerically sampling the mode, using an implementation of the recursion (23) in Mathematica, we find that the mode increases rapidly for large $\theta$ and small $\gamma$, see also figure 5 . 


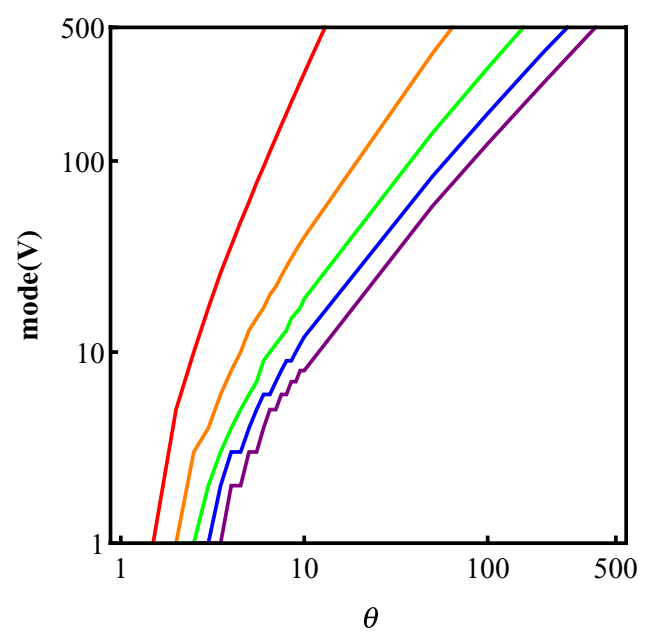

Figure 5. The mode of $V$ plotted as function of $\theta$ and $q=0.5$. We chose (beginning from the left) $\gamma$ equal to $0.5,1,1.5,2$ and 2.5. The jagged appearance of some of the lines is due to the mode taking only integer values.

The probability $p_{0}$ is indeed an important quantity in itself, since the 'resistance probability' $\mathbb{P}(V>0)=1-p_{0}$ indicates, in the sense of the original Luria-Delbrück formulation, the probability that a population can escape extinction under the attack of a lethal virus due to the existence of resistant mutants. Thanks to the recursion (23), we can give $p_{0}$ explicitly

$$
\mathbb{P}(V=0)=\exp \left[-\frac{\theta}{\gamma} F\left(\begin{array}{c}
1, \gamma \\
1+\gamma
\end{array} ; q\right)\right] .
$$

The expression $1-p_{0}$, derived differently, appears in $[14,(7)]$ (Note that they have another definition of the mutation rate, which maps into ours with $\delta=(1-u) r$ and $\nu=u r$, where $u$ is the mutation probability). With increasing mode the resistance probability increases, in fact exponentially fast by (28). For $\gamma \rightarrow \infty$ and fixed $\theta$ the resistance probability goes to 0 . For $\gamma$ fixed and $\theta \rightarrow \infty$, the probability goes to one. Using a Taylor expansion of the hypergeometric function in (28), we can approximate the resistance probability for large $\gamma$ as

$$
-\log p_{0} \sim \frac{\theta}{\gamma(1-q)+q}
$$

For a fixed $p_{0}$, the two variables $\theta$ and $\gamma$ can be seperated and the contour associated to the fixed value of $p_{0}$ can be parameterized as

$$
\theta \sim-[\gamma(1-q)+q] \log p_{0} .
$$

A plot of the contours, where the resistance probability of $V$ is equal to $1 / 2$ for different $q$ is given in figure 6 together with their approximations.

\subsection{Large values of $\theta$}

We now let $\theta \rightarrow \infty$, using the results of the previous section. Obviously, for $\theta \rightarrow \infty$ (21) does not converge, thus we need to introduce a scaling. We formalize this convergence in 


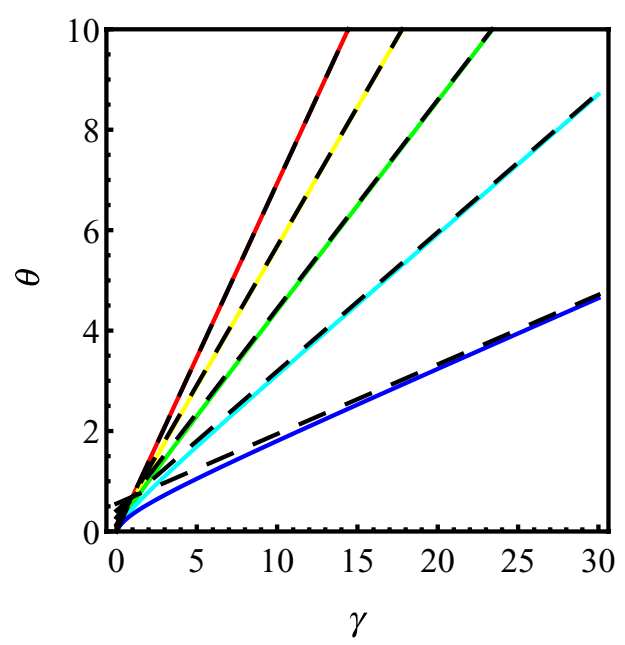

Figure 6. Plot of the contours dependend of $\gamma$ and $\theta$ where the probability $p_{0}=1 / 2$ for $q=0,0.2,0.4,0.6,0.8$ (left to right) and the approximation (dashed line) given in (5.1). Note that the quality of the approximation depends on $q$.

distribution by

$$
\lim _{\theta \rightarrow \infty} \frac{V}{a}-b=Z
$$

where $a, b$ depend only on $\theta$. The scaling factor is in fact already predetermined, see $[28$, section 2.7], and is proportional to $\theta^{1 / \min (\gamma, 2)}$, but we will re-derive it for our case. We take the opportunity to scale out the survival probability $1-q$ wherever possible.

Let us abbreviate the logarithm of the Laplace transform of $V$ by

$$
\mathcal{E}_{V}(s)=\log \mathbb{E}(\exp (-V s))=\Lambda_{V}\left(\mathrm{e}^{-s}\right) .
$$

This definition directly implies

$$
\mathcal{E}_{\frac{V}{a}-b}(s)=\mathcal{E}_{V}(s / a)+b s .
$$

We use this notation also for $W$.

For $\left.y\right|_{z=\mathrm{e}^{-s / a}}$, equation (21) turns into

$$
\mathcal{E}_{V}(s / a)=-\frac{\theta}{\gamma} F\left(\begin{array}{c}
1, \gamma \\
1+\gamma
\end{array} ; \frac{\mathrm{e}^{-s / a}-q}{\mathrm{e}^{-s / a}-1}\right) .
$$

We then apply equation (C.13) to derive

$$
\mathcal{E}_{V}(s / a)=\frac{\theta\left(\mathrm{e}^{-s / a}-1\right)}{(1-q)(\gamma-1)} F\left(\begin{array}{c}
1,1 \\
2-\gamma
\end{array} ; \frac{1-\mathrm{e}^{-s / a}}{1-q}\right)+\frac{\pi}{\sin (\pi \gamma)}\left(-\frac{\theta^{1 / \gamma}\left(\mathrm{e}^{-s / a}-1\right)}{\mathrm{e}^{-s / a}-q}\right)^{\gamma} \text {. }
$$

For $a$ to infinity $\mathrm{e}^{-s / a}-q \rightarrow 1-q$ and $F\left({ }_{2-\gamma}^{1,1} ; \frac{1-\mathrm{e}^{-s / a}}{1-q}\right) \rightarrow F\left({ }_{2-\gamma}^{1,1} ; 0\right)=1$ for all $\gamma>0$. With a Taylor expansion of $\mathrm{e}^{-s / a}$ around $s=0$ we can write

$\mathcal{E}_{V}(s / a) \sim \sum_{k \geqslant 1} \frac{(-s)^{k}}{\theta^{-1} a^{k}(1-q)(\gamma-1) k !}+\frac{\pi}{\sin (\pi \gamma)}\left(\sum_{k \geqslant 1} \frac{(-s)^{k}}{\theta^{-1 / \gamma} a^{k}(1-q) k !}\right)^{\gamma}$.

For the computations we consider four cases, which depend on the choice of $\gamma$. The results are listed in table 2. Note, that although the expressions for $\gamma \in(0,1)$ and $\gamma \in(1,2)$ are 
Table 2. Overview or the results of the large $\theta$ limit. Note that although the expressions in the first and third row are identical, the means are not.

\begin{tabular}{llll}
\hline$\gamma$ & $\mathcal{E}_{Z}(s)$ & $\mathbb{E}(Z)$ & $\operatorname{Var}(Z)$ \\
\hline$\gamma \in(0,1)$ & $\frac{\pi}{\sin (\pi \gamma)} s^{\gamma}$ & $+\infty$ & $+\infty$ \\
$\gamma=1$ & $s \log s$ & $+\infty$ & $+\infty$ \\
$\gamma \in(1,2)$ & $\frac{\pi}{\sin (\pi \gamma)} s^{\gamma}$ & 0 & $+\infty$ \\
$\gamma \geqslant 2$ & $\frac{1}{2} s^{2}$ & 0 & 1 \\
\hline
\end{tabular}

equal, the means are not. Indeed this can be understood via a derivative with respect to $s$ and a limit $s \rightarrow 0$.

$\gamma>2$. For $\gamma>2$ it is now intuitive to set $a=\sqrt{\frac{\theta}{(\gamma-1)(1-q)}}$, since for this choice all terms except the linear and quadratic term in the first addend of (31) vanish for $\theta \rightarrow \infty$. The linear term however diverges, so we compensate it by setting $b=\sqrt{\frac{\theta}{(1-q)(\gamma-1)}}$. Thus

$$
\mathcal{E}_{Z}(s)=\lim _{\theta \rightarrow \infty} \mathcal{E}_{V}(s / a)+b s=\frac{s^{2}}{2},
$$

which proofs that the limit random variable $Z$ has a standard normal distribution.

$0<\gamma<2, \gamma \neq 1$. In this case it is sufficient to set $a=\theta^{1 / \gamma} /(1-q)$, then (31) is

$$
\mathcal{E}_{V}(s / a) \sim-\frac{\theta^{1-1 / \gamma}}{\gamma-1} s+O\left(\theta^{1-2 / \gamma}\right)+\frac{\pi}{\sin (\pi \gamma)}\left[s+O\left(\theta^{-1 / \gamma}\right)\right]^{\gamma} .
$$

Therefore, if we set $b=\frac{\theta^{1-1 / \gamma}}{\gamma-1}$,

$$
\mathcal{E}_{Z}(s)=\lim _{\theta \rightarrow \infty} \mathcal{E}_{V}(s / a)+b s=\frac{\pi}{\sin (\pi \gamma)} s^{\gamma} .
$$

Note that for $0<\gamma<1$, we could have chosen $b=0$, since $\frac{\theta^{1-1 / \gamma}}{\gamma-1} \rightarrow 0$. The result resembles and in fact generalizes the result of [17, theorem 6] and [18].

$\gamma=1$. Here we have to consider (30), since the expansion (31) has a singularity at $\gamma=1$. We use (C.17) instead and gain

$$
\mathcal{E}_{V}(s / a)=\frac{\theta\left(\mathrm{e}^{-s / a}-1\right)}{\mathrm{e}^{-s / a}-q}\left[\log \left(\frac{\theta\left(\mathrm{e}^{-s / a}-1\right)}{q-1}\right)-\log \theta\right] .
$$

With the same argumentation of Taylor expansion of $\theta\left(\mathrm{e}^{-s / a}-1\right)$ as in the proceeding cases we notice that $a=\theta /(1-q)$ is a sensible choice, since then

$$
\mathcal{E}_{V}(s / a) \sim s \log s-s \log \theta
$$

and consequently for $b=\log \theta$

$$
\mathcal{E}_{Z}(s)=\lim _{\theta \rightarrow \infty} \mathcal{E}_{V}(s / a)+b s=s \log s,
$$

which also appears in [19]. 
$\gamma=2$. Here we face the same problem as in the previous case, since the expansion (31) is also singular at $\gamma=2$. Via consecutive application of (30) and (C.18) we gain

$$
\mathcal{E}_{V}(s / a)=\frac{\theta\left(\mathrm{e}^{-s / a}-1\right)}{\mathrm{e}^{-s / a}-a}-\frac{\left[\theta\left(\mathrm{e}^{-s / a}-1\right)\right]^{2}}{\theta\left(\mathrm{e}^{-s / a}-q\right)^{2}} \log \left(\frac{\theta \mathrm{e}^{-s / a}-1}{\theta(q-1)}\right) .
$$

With the very same argumentation as in the $\gamma=1$ case, we find for $a=\frac{\sqrt{\theta \log \theta}}{1-q}$

$$
\mathcal{E}_{V}(s / a) \sim-s \sqrt{\frac{\theta}{\log \theta}}+\frac{\log (\theta \log \theta)}{2 \log \theta} s^{2},
$$

so that with $b=\sqrt{\frac{\theta}{\log \theta}}$

$$
\mathcal{E}_{Z}(s)=\lim _{\theta \rightarrow \infty} \mathcal{E}_{V}(s / a)+b s=\frac{1}{2} s^{2} .
$$

Again, $Z$ has a standard normal distribution.

\subsection{Large population limit at fixed mutation rate}

In this section we extend the results of Mandelbrot from [18] to allow the possibility of mutant death. We consider a similar approach as in the large $\theta$-limit but with mutation rate $\nu$ held constant, i.e.

$$
\lim _{N \rightarrow \infty} \frac{B}{a}-b=W
$$

where $a$ and $b$ depend on $N$. Like in (5.2), we abbreviate with $\mathcal{E}_{B}(s)$ the log-Laplace transform of $B$ and note that

$$
\mathcal{E}_{\frac{B}{a}-b}(s)=\mathcal{E}_{B}(s / a)+b s .
$$

Applying first (C.13) and then (C.10) to (7) gives, noticing that $\mathrm{e}^{-s / a}-q \rightarrow 1-q$,

$$
\begin{aligned}
\mathcal{E}_{B}(s / a) \sim & \frac{\mu}{(\gamma-1)}\left[-\frac{N^{1 / \gamma}\left(\mathrm{e}^{-s / a}-1\right)}{1-q} F\left(\begin{array}{c}
1,1-\gamma \\
2-\gamma
\end{array} ; \frac{N^{1 / \gamma}\left(\mathrm{e}^{-s / a}-1\right)}{1-q}\right)\right. \\
& \left.+\frac{N\left(\mathrm{e}^{-s / a}-1\right)}{1-q} F\left(\begin{array}{c}
1,1-\gamma \\
2-\gamma
\end{array} ; \frac{\mathrm{e}^{-s / a}-1}{1-q}\right)\right] .
\end{aligned}
$$

The parameter $a$ controls the variance, therefore we know that we should choose $a$ of the order of $\sqrt{\operatorname{Var}(B)}$. The parameter $b$ should be proportional to the order of the ratio $\mathbb{E}(B) / \sqrt{\operatorname{Var}(B)}$, see also figure 1 . The limits can now be computed like in the LPSMlimit by expanding $\mathrm{e}^{-s / a}$ into a power series. We give the chosen scaling factors together with the limit results and their mean and variance in table 3. Note that we could have removed the mean for $\gamma \in(0,1]$, but we need a non-zero expectation for the $\mu \rightarrow 0$ limit to recover $Z$.

In order to check that the two pathways for going from $B$ to $Z$ on figure 2 are equivalent, we take the $\mu \rightarrow 0$ limit of the above large $N$ limit. Again, we consider the limit

$$
\lim _{\mu \rightarrow 0} \frac{W}{a}=Z
$$

where we assume for simplicity that $a$ is now only dependent on $\mu$. 
Table 3. Overview of the limit results for $N \rightarrow \infty$ and $\mu=$ const.

\begin{tabular}{llllll}
\hline & $(1-q) a$ & $b$ & $\mathcal{E}_{W}(s)$ & $\mathbb{E}(W)$ & $\operatorname{Var}(W)$ \\
\hline$\gamma>2$ & $\sqrt{N}$ & $\frac{\mu}{\gamma-1} \sqrt{N}$ & $\frac{1}{2} \mu \frac{\gamma-q(\gamma-2)}{(\gamma-2)(\gamma-1)} s^{2}$ & 0 & $\frac{\mu(\gamma-q(\gamma-2))}{(\gamma-2)(\gamma-1)}$ \\
$\gamma=2$ & $\sqrt{N \log N}$ & $\mu \sqrt{\frac{N}{\log N}}$ & $\mu \frac{s^{2}}{2}$ & 0 & $\mu$ \\
$\gamma \in(1,2)$ & $N^{1 / \gamma}$ & $\frac{\mu}{\gamma-1}\left(N^{1-\frac{1}{\gamma}-1}\right)$ & $\frac{\mu}{2-\gamma} s^{2} F\left(\begin{array}{c}1,2-\gamma \\
3-\gamma\end{array} ;-s\right)$ & 0 & $\frac{2 \mu}{2-\gamma}$ \\
$\gamma=1$ & $N$ & $\mu(1+\log N)$ & $\mu s(1+\log (1+s))$ & $\mu$ & $2 \mu$ \\
$\gamma \in(0,1)$ & $N^{1 / \gamma}$ & 0 & $\frac{\mu}{\gamma-1} s F\left(\begin{array}{c}1,1-\gamma \\
2-\gamma\end{array} ;-s\right)$ & $\frac{\mu}{\gamma-1}$ & $\frac{2 \mu}{2-\gamma}$ \\
\hline
\end{tabular}

For $\gamma \geqslant 2$ there is nothing to show, since for any $X \sim \mathcal{N}\left(\mu, \sigma^{2}\right)$ obviously $\frac{X-\mu}{\sigma} \sim$ $\mathcal{N}(0,1)$. Therefore $\frac{W-\mathbb{E}(W)}{\operatorname{Var}(W)}$ does not depend on $\mu$ and the limit for $\mu \rightarrow 0$ is trivially again a Gaussian. We notice that by using (C.19) in reverse

$$
\frac{\mu}{2-\gamma} s^{2} F\left(\begin{array}{c}
1,2-\gamma \\
3-\gamma
\end{array} ;-s\right)=\frac{\mu}{1-\gamma} s-\frac{\mu}{1-\gamma} s F\left(\begin{array}{c}
1,1-\gamma \\
2-\gamma
\end{array} ;-s\right)
$$

and by equation (C.13) we get

$$
\frac{\mu}{1-\gamma} s F\left(\begin{array}{c}
1,2-\gamma \\
3-\gamma
\end{array} ;-s\right)=\frac{\pi}{\sin (\pi \gamma)} \mu s^{\gamma}-\frac{s \mu}{(s+1) \gamma} F\left(\begin{array}{c}
1,1 \\
1+\gamma
\end{array} ; \frac{1}{1+s}\right) .
$$

Finally, setting $a=\mu^{1 / \gamma}$, we get

$$
\mathcal{E}_{Z}(s)=\lim _{\mu \rightarrow 0} \mathcal{E}_{W}(s / a)= \begin{cases}s \log s & \gamma=1 \\ \frac{\pi}{\sin (\pi \gamma)} s^{\gamma} & \gamma \in(0,2) \backslash\{1\}\end{cases}
$$

That shows that the scaling limit of $W$ for $\mu \rightarrow 0$ recovers indeed the distributions we got for the large $\theta$ limit, which we give in table 2 .

\subsection{On $\alpha$-stable distributions}

We point out that the limiting Laplace transforms given in table 2 are well known representatives of the class of $\alpha$-stable distributions, where $\alpha \in(0,2]$. These distributions appear as the limit distributions in the generalized version of the Central Limit Theorem, where iid. random variables are added and rescaled, but the assumption of finite mean and variance is dropped. The Gaussian distribution is in this context the extreme case for $\alpha=2$ and the only limit distribution in this class with finite variance. As a reference see [28, section 2.7].

One of the many characterizations of $\alpha$-stable distributions is given via characteristic functions (the notation varies strongly throughout the literature). A random variable $X$ with characteristic function

$$
\varphi_{X}(s)=\mathbb{E}(\exp (\mathrm{i} X s))
$$


is $\alpha$-stable respectively $X \sim S_{\alpha}(\sigma, \beta, \mu)$ if and only if

$$
\log \varphi_{X}(s)= \begin{cases}-\sigma^{\alpha}|s|^{\alpha}\left(1-\mathrm{i} \beta \operatorname{sgn}(s) \tan \frac{\alpha \pi}{2}\right)+\mathrm{i} \mu s & \alpha \neq 1 \\ -\sigma|s|\left(1+\mathrm{i} \beta \frac{2}{\pi} \operatorname{sgn}(s) \log s\right)+\mathrm{i} \mu s & \alpha=1 .\end{cases}
$$

By a formal substitution $s \mapsto-$ is, we can rewrite the distribution of $Z$, as given in table 2 using the above notation

$$
\log \varphi_{Z}(s)= \begin{cases}-\sigma^{\gamma}|s|^{\gamma}\left(1-\mathrm{i} \operatorname{sgn}(s) \tan \left(\frac{\pi \gamma}{2}\right)\right) & \gamma \in(0,2) \backslash\{1\} \\ -\frac{\pi}{2}|s|\left(1+\mathrm{i} \operatorname{sgn}(s) \frac{2}{\pi} \log s\right) & \gamma=1 \\ -\frac{1}{2} s^{2} & \gamma \geqslant 2,\end{cases}
$$

where

$$
\sigma=\left[\frac{\pi \gamma}{2} \csc \left(\frac{\pi \gamma}{2}\right)\right]^{1 / \gamma}
$$

This means that $Z$ is indeed an $\alpha$-stable random variable for all $\gamma>0$, where the shapeparameter $\alpha=\min (\gamma, 2)$.

Unfortunately, the densities are unknown for the majority of $\alpha$-stable distributions. One exception is the Gaussian distribution $(\alpha=2)$. The Lévy-Distribution $S_{1 / 2}(\sigma, 1,0)$ with density

$$
f(t)=\left(\frac{\sigma}{2 \pi}\right)^{1 / 2} \frac{1}{t^{3 / 2}} \exp \left(-\frac{\sigma}{2 t}\right)
$$

and infinite moments and the Holtsmark distribution with finite mean are other cases (for the latter a lengthy expression in terms of hypergeometric functions exists). In the special case for $\gamma=1$, we find that $Z$ is characterized by the distribution $S_{1}(\sigma, 1,0)=$ $\left.S_{1}(\pi / 2,1,0)\right)$, which is the Landau-Distribution. This distribution was also identified for the fully stochastic case by Kessler and Levine in [2].

\section{Tail behavior}

The mutant distributions as we described them in the previous section 5 are quite complicated. For this reason we study their tail behavior to gain some intuition. We use asymptotic analysis methods as discussed in [29].

We start with the most interesting case, the LPSM limit, where the number of mutants, denoted by $V$ (see figure 2), is characterized by generating function (22). The tail behavior of $p_{n}=P(V=n)$ is encoded in $G(z)$ around its relevant (closest to origin) singularity. This generating function is analytic at the origin, and its only singularity is at $z=1$, which implies $y=\frac{z-q}{1-q}=1$, so we expand around that. We first use the transformation formula (C.14) and then the reflection formulas (C.1) and (C.2) to rewrite the log-generating function as

$$
\Lambda(z)=\theta \frac{1-y}{1-\gamma} F\left(\begin{array}{c}
1,1 \\
2-\gamma
\end{array} ; 1-y\right)-\frac{\theta \pi}{\sin (\gamma \pi)} y^{-\gamma}(1-y)^{\gamma} .
$$


An expansion around $y=1$ gives

$$
\begin{aligned}
\Lambda(z)=\frac{\theta}{1-\gamma} & {\left[(1-y)+\frac{(1-y)^{2}}{2-\gamma}+O\left((1-y)^{3}\right)\right] } \\
& -\frac{\theta \pi}{\sin (\gamma \pi)}\left[(1-y)^{\gamma}+\gamma(1-y)^{1+\gamma}+O\left((1-y)^{2+\gamma}\right)\right],
\end{aligned}
$$

and by using the series expansion of the exponential we get

$$
\begin{aligned}
G(z)=\mathrm{e}^{\Lambda(z)}= & 1+\Lambda(z)+\frac{\Lambda^{2}(z)}{2}+O\left(\Lambda^{3}(z)\right) \\
= & K(1-y)-\kappa(1-y)^{\gamma}-\kappa\left(\frac{\theta}{1-\gamma}+\gamma\right)(1-y)^{1+\gamma}+\frac{1}{2} \kappa^{2}(1-y)^{2 \gamma} \\
& +O\left((1-y)^{\{3 \gamma, 2+\gamma, 1+2 \gamma\})},\right.
\end{aligned}
$$

where $K$ is a polynomial with integer powers (hence does not contribute to the tail behavior), and for brevity we wrote

$$
\kappa=\frac{\theta \pi}{\sin (\gamma \pi)} .
$$

Note that this is already an expansion in $1-z$ since

$$
1-y=\frac{1-z}{1-q}
$$

By using (D.1) and (C.1) we obtain the large $n$ expansion

$$
\begin{aligned}
p_{n} \sim \frac{\theta \Gamma(1+\gamma)}{(1-q)^{\gamma}} & n^{-1-\gamma}+\frac{\theta^{2} \kappa^{2}}{2 \Gamma(-2 \gamma)(1-q)^{2 \gamma}} n^{-1-2 \gamma} \\
& -\frac{\theta \Gamma(2+\gamma)}{(1-q)^{1+\gamma}}\left(\frac{\theta}{1-\gamma}+\frac{\gamma(1+q)}{2}\right) n^{-2-\gamma}+O\left(n^{\{3 \gamma, 2+\gamma, 1+2 \gamma\}}\right) .
\end{aligned}
$$

The leading order term has been derived in [2]. The sub leading order is represented by the $n^{-1-2 \gamma}$ term for $\gamma<1$, and by the $n^{-2-\gamma}$ term for $\gamma>1$.

Let us consider the above expansion at the special value $\gamma=1$. As $\gamma \rightarrow 1$, both subleading terms in (33) diverge as $\propto 1 /|1-\gamma|$ but these two singular terms cancel each other out, and one has to consider the next (constant) terms in the $(1-\gamma)$ series. A logarithmic term appears through the expansion

$$
n^{1-\gamma}=\mathrm{e}^{(1-\gamma) \log n}=1+(1-\gamma) \log n+O[(1-\gamma) \log n]^{2}
$$

for $\gamma \rightarrow 1$. We arrive at

$p_{n} \sim \frac{\theta}{(1-q) n^{2}}+\frac{2 \theta^{2}}{(1-q)^{2}} \frac{\log n}{n^{3}}+\frac{\theta^{2}\left[2 C_{\mathrm{E}}-3-2 L(q)\right]-\theta(1+q)}{(1-q)^{2} n^{3}}+O\left(\frac{1}{n^{4}}\right)$

where $L(z)=\log \frac{1}{1-z}$. For $q \rightarrow 0$, we have $L(q) \rightarrow 0$, and we obtain

$$
p_{n}=\frac{\theta}{n^{2}}+2 \theta^{2} \frac{\log n}{n^{3}}+\frac{\theta^{2}\left(2 C_{\mathrm{E}}-3\right)-\theta}{n^{3}}+3 \theta^{3} \frac{\log ^{2} n}{n^{4}}+O\left(\frac{\log n}{n^{4}}\right)
$$

where $C_{\mathrm{E}}=0.5772 \ldots$ is the Euler-Mascheroni constant. The first three terms have been calculated in [24]. Formally, all terms can be included, and here we just give the result

$$
\begin{aligned}
p_{n} & =\sum_{k=1}^{\infty} \frac{\theta^{k}}{k !(n+k) !} \lim _{r \rightarrow k} \partial_{r}^{k} \frac{\Gamma(n+k-r)}{\Gamma(-r)} \\
& =\frac{\theta}{n(n+1)}+\frac{\theta^{2}\left[2 C_{\mathrm{E}}-3+2 \Psi(n)\right]}{n(n+1)(n+2)}+O\left(\frac{\theta^{3} \log ^{2} n}{n^{4}}\right) .
\end{aligned}
$$


This expression is exact for any specific order of $\theta$. By expanding it around $n=\infty$, using the expansion of the Digamma function $\psi(n)$ given in (C.4), we recover (35).

The tail behavior of the full distribution of $B$ with finite $N$ is similar, but additionally it has an exponential cut-off. We demonstrate this behavior only for the $\gamma=1$ and $q=0$ case for simplicity. In this case the generating function is given by (17), which is again

$$
G(z)=(1-\phi z)^{\theta \frac{1-z}{z}}
$$

with $\phi=1-1 / N$. Now the singularity is at $z=1 / \phi$, which leads to an exponential cut-off since

$$
\left[z^{n}\right] G(z)=\phi^{n}\left[z^{n}\right] G(z / \phi)
$$

where $\left[z^{n}\right]$ means the coefficient of $z^{n}$ in the Taylor expansion of the subsequent expression. We now write

$$
G(z / \phi)=(1-z)^{\theta \frac{\phi-z}{z}}=(1-z)^{-\mu}(1-z)^{(\theta-\mu) \frac{1-z}{z}} .
$$

The second factor is analytic at $z=0$, and its only singularity is at $z=1$ (and a branch cut from $z=1$ to infinity). Here the exponent approaches zero, so we expand first the exponential function

$$
\begin{aligned}
G(z / \phi) & =\sum_{k \geqslant 0} \frac{(\mu-\theta)^{k}}{k !}(1-z)^{k-\mu}\left(\frac{L}{z}\right)^{k} \\
& =(1-z)^{-\mu}+(\mu-\theta) \frac{(1-z)^{1-\mu}}{z} L+\ldots
\end{aligned}
$$

with $L \equiv L(z)=\log \frac{1}{1-z}$. Note that we did not expand $1 / z$ around $z=1$, and the reason for that becomes clear in the next step. We obtain the coefficients by using (D.1) and the Frobenius-Jungen method (D.5) from [29]

$$
\begin{aligned}
{\left[z^{n}\right] G(z / \phi) } & =\sum_{k \geqslant 0} \frac{(\mu-\theta)^{k}}{k !}\left[z^{n+k}\right](1-z)^{k-\mu} L^{k} \\
& =\frac{n^{\mu-1}}{\Gamma(\mu)}\left(1+\sum_{k \geqslant 1} \frac{e_{k}}{n^{k}}\right)+\left.\sum_{k \geqslant 1} \frac{(\mu-\theta)^{k}}{k !} \frac{\mathrm{d}^{k}}{\mathrm{~d} a^{k}}\left(\begin{array}{c}
n+k+a-1 \\
n+k
\end{array}\right)\right|_{a=n-k}
\end{aligned}
$$

The first few terms are

$$
\begin{aligned}
{\left[z^{n}\right] G(z / \phi)=} & \frac{n^{\mu-1}}{\Gamma(\nu)}\left[1+\frac{\mu(\mu-1)}{2} n^{-1}+O\left(n^{-2}\right)\right] \\
& +(\mu-\theta)\left(\begin{array}{c}
n+\mu-1 \\
n+1
\end{array}\right)[\Psi(\mu-1+n)-\Psi(\mu-1)]+O\left(n^{\mu-3} \log n\right)
\end{aligned}
$$

and the leading powers of $n$ are

$$
\left[z^{n}\right] G(z / \phi)=\frac{n^{\mu-1}}{\Gamma(\mu)}+\frac{n^{\mu-2}}{\Gamma(\mu-1)}\left\{(\mu-\theta)[\log n-\Psi(\mu-1)]+\frac{\mu}{2}\right\}+O\left(n^{\mu-3} \log n\right) .
$$

Now we also include the exponential cut-off from (37) to obtain

$$
\begin{aligned}
& p_{n} \sim \frac{(1-1 / N)^{n}}{\Gamma(\mu)}\left\{\frac{1}{n^{1-\mu}}+(1-\mu)(\theta-\mu) \frac{\log n}{n^{2-\mu}}\right. \\
&\left.-(1-\mu)\left[(\theta-\mu) \Psi(\mu-1)+\frac{\mu}{2}\right] \frac{1}{n^{2-\mu}}+O\left(\frac{\log n}{n^{3-\mu}}\right)\right\} .
\end{aligned}
$$


Mutant number distribution in an exponentially growing population

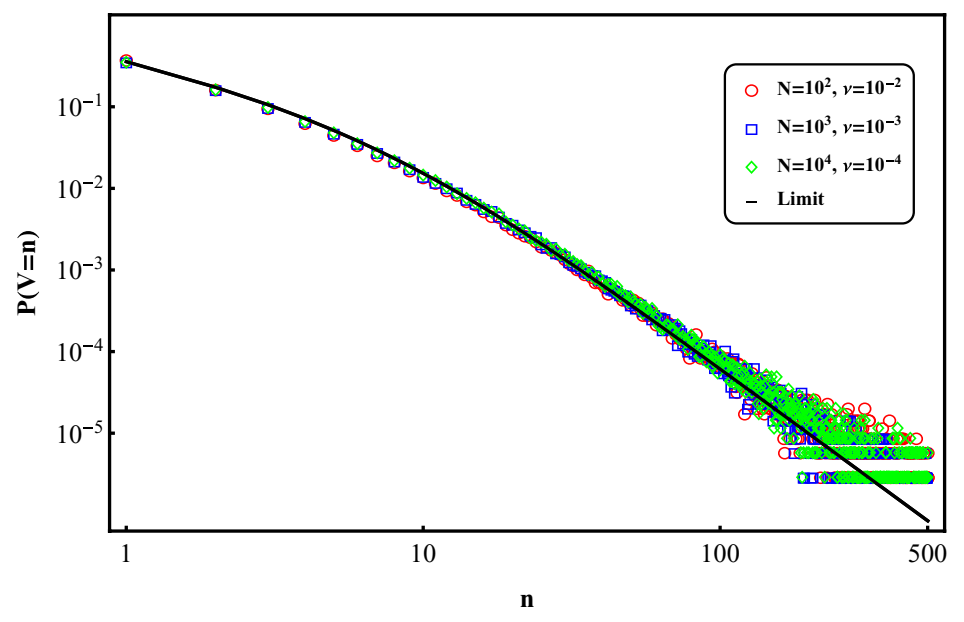

Figure 7. Simulation of the fully stochastic model against the $N \mu=1$-limit $(\alpha=1, \delta=1.0, \lambda=1.3)$ for $10^{6}$ trajectories each.

The leading term of this expansion has been derived in [23]. Also, if we take the $\mu \rightarrow 0, N \rightarrow \infty$ limit of this asymptotic expansion, noting that $\Gamma(\mu) \sim 1 / \mu$ and $\Psi(\mu-1) \sim-1 / \mu$ as $\mu \rightarrow 0$, we recover the small mutation expression (35).

\section{Discussion and summary}

We revisited a well-known semi-stochastic model with exponential wild-type growth and mutants that evolve according to a supercritical birth and death process. We give an explicit expression for the log-generating function $\Lambda_{B}(z)$ of this process in terms of hypergeometric functions, see equation (7). This allows us to compute limits and probabilities. We recover the limit results by Kessler and Levine in [2] and [16], Durrett and Moseley [17], Mandelbrot [18] and Möhle [19], and extend them to a wider range of parameters and include mutant-cell death, which is not treated in every case.

To emphasize that the semi-deterministic model has many advantages over the fully stochastic one, we compared simulation results of the fully stochastic model with the results of the LPSM-limit taken in section 5, see figures 7 and 8. We find good agreement between simulation results and the semi-stochastic theory already for relatively small values of $N$.

We showed that the mutant distribution depends on the mutant extinction probability $q=\beta / \alpha$ in a nontrivial way, and it cannot simply be scaled out of the formulas. In the large $\theta=N \mu$ limit, however, $q$ can be scaled out of the mutant distribution for $\gamma \in(0,2]$, hence only in this limit cell death has a trivial effect on the mutant distribution.

From numerical work we found that the mutant distribution is unimodal, i.e. it has only a single maximum. We gave a simple condition for having no mutants being the most probable scenario. Otherwise, the most probable number of mutants is positive, which we determined numerically and plotted for several parameter values.

We also determined the mean and the variance of the number of mutants, which are of course finite for finite wild-type population size, $N$. When taking the large $N$ 


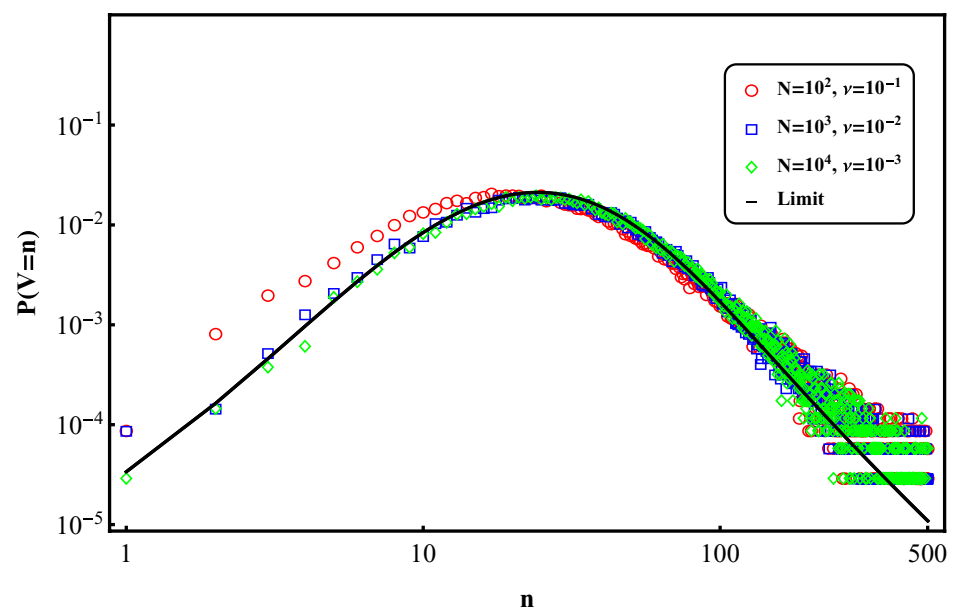

Figure 8. Simulation of the fully stochastic model against the $N \mu=10$-limit $(\alpha=1, \delta=1.0, \lambda=1.3)$ for $10^{6}$ trajectories each.

limit and using the appropriate scaling, we also obtain finite moments. Conversely, in the large-population-small-mutation limit the moments become infinite for $\gamma \in[0,2]$. This behavior, which caused some controversy in the literature (see [5] for historical notes on the topic), is explained by the power law tail of the mutant distribution in this limit.

\section{Acknowledgments}

We thank the National Philantropic Trust (FQEB Grant \#RFP-12-18) for financial support and the anonymous referee for valuable input.

\section{Appendix A. Proof of recursion formulas}

To prove the recursion (10) we first Taylor expand $\Lambda_{B}(z)$ around $z=0$ as

$$
\Lambda_{B}(z)=\sum_{n=0}^{\infty} q_{n} z^{n}
$$

Equating the coefficients of $z^{n}$ on both sides of

$$
G(z)=\sum_{n=0}^{\infty} p_{n} z^{n}=\mathrm{e}^{\sum_{n}^{\infty} q_{n} z^{n}}
$$

leads to the general recursion (10) (stated as lemma 2 in [5]).

When Taylor expanding $\Lambda_{B}(z)$, we immediately obtain $q_{0}$ by replacing $\xi$ by $q=\left.\xi\right|_{z=0}$ in (7). To obtain higher order coefficients, we first calculate the coefficients $a_{k}$ of the expansion of

$$
\frac{1}{\gamma} F\left(\begin{array}{c}
1, \gamma \\
1+\gamma
\end{array} ; N^{-1 / \gamma} \xi\right)=\sum_{k \geqslant 0} a_{k} z^{k}
$$


With induction one can show that for $k \geqslant 1$

$$
\begin{aligned}
\frac{\mathrm{d}^{k}}{\mathrm{~d} z^{k}} F\left(\begin{array}{c}
1, \gamma \\
1+\gamma
\end{array} ; N^{-1 / \gamma} \xi\right) \\
\quad=k ! \sum_{j=1}^{k}\left(\begin{array}{c}
k-1 \\
j-1
\end{array}\right) \frac{\gamma(q-1)^{j} N^{-j / \gamma}}{(\gamma+j)(1-z)^{j+k}} F\left(\begin{array}{c}
1+j, \gamma+j \\
1+\gamma+j
\end{array} ; N^{-1 / \gamma} \xi\right) .
\end{aligned}
$$

Here the $k=1$ case can be easily checked, and the induction can be performed by differentiating the above expression using (C.14). Now we take (A.4) at $z=0$ and simplify it by using (C.11) to find the coefficients

$$
a_{k}=\sum_{j=1}^{k}\left(\begin{array}{c}
k-1 \\
j-1
\end{array}\right) \frac{1}{j+\gamma}\left(\frac{1-q}{q-N^{1 / \gamma}}\right)^{j} F\left(\begin{array}{c}
1, \gamma \\
1+\gamma+j
\end{array} ; N^{-1 / \gamma} q\right) .
$$

For the second term in (7) we need the $N=1$ case, where the above sum simplifies

$$
\begin{aligned}
a_{k} & =\sum_{j=1}^{k}\left(\begin{array}{l}
k-1 \\
j-1
\end{array}\right) \frac{(-1)^{j}}{j+\gamma} F\left(\begin{array}{c}
1, \gamma \\
1+\gamma+j
\end{array} ; q\right) \\
& =\sum_{n \geqslant 0} q^{n}(\gamma)_{n} \sum_{j=1}^{k}\left(\begin{array}{l}
k-1 \\
j-1
\end{array}\right) \frac{(-1)^{j}}{(j+\gamma)_{n+1}} .
\end{aligned}
$$

We can calculate the second sum by first generalizing it to a polynomial, re-indexing it, and realizing that we can extend the summation to infinity

$$
\begin{aligned}
(\gamma)_{n} \sum_{j=1}^{k}\left(\begin{array}{c}
k-1 \\
j-1
\end{array}\right) \frac{(-1)^{j} z^{j-1}}{(j+\gamma)_{n+1}} \\
=-(\gamma)_{n} \sum_{j \geqslant 0}\left(\begin{array}{c}
k-1 \\
j
\end{array}\right) \frac{(-1)^{j} z^{j}}{(j+\gamma+1)_{n+1}} \\
=-\frac{\gamma}{(\gamma+n)(\gamma+n+1)} F\left(\begin{array}{c}
1-k, 1+\gamma \\
2+n+\gamma
\end{array} ; z\right) .
\end{aligned}
$$

The parameters of the hypergeometric function were read out from the ratio of consecutive coefficients of the series, as in (C.7). Now taking the $z / 1$ limit in (A.8) and using the $\mathrm{Chu}$-Vandermonde identity (C.9) yields

$$
(\gamma)_{n} \sum_{j=1}^{k}\left(\begin{array}{c}
k-1 \\
j-1
\end{array}\right) \frac{(-1)^{j}}{(j+\gamma)_{n+1}}=-\gamma \frac{(n+1)_{k-1}}{(n+\gamma)_{k+1}}
$$

We can substitute this expression into (A.6) to obtain

$$
a_{k}=-\gamma \sum_{n \geqslant 0} q^{n} \frac{(n+1)_{k-1}}{(n+\gamma)_{k+1}}=-\frac{(k-1) !}{(\gamma+1)_{k}} F\left(\begin{array}{c}
k, \gamma \\
1+\gamma+k
\end{array} ; q\right),
$$

which immediately leads to (12).

For the special case $\gamma=1$ we present an easier derivation. We derive a recursion for the probabilities $p_{n}$ directly from the expression (16) by Taylor expanding around $z=0$ and using the binomial theorem to rewrite $y^{j}$ as

$$
y^{j}=(1-q)^{-j} \sum_{k=0}^{j}\left(\begin{array}{l}
j \\
k
\end{array}\right)(-q)^{j-k} z^{k} .
$$


Then

$$
\begin{aligned}
\Lambda_{B}(z) & =\theta \frac{1-y}{y} \log (1-\phi y) \\
& =-\theta \phi+\theta \sum_{j \geqslant 1}\left(\frac{\phi^{j}}{j}-\frac{\phi^{j+1}}{j+1}\right) y^{j} \\
& =-\theta \phi+\theta \sum_{j \geqslant 1} \sum_{k=0}^{j}\left(\begin{array}{l}
j \\
k
\end{array}\right) \frac{(-q)^{j-k}}{(1-q)^{j}}\left(\frac{\phi^{j}}{j}-\frac{\phi^{j+1}}{j+1}\right) z^{k} .
\end{aligned}
$$

By changing the order of summation, we read out the coefficients $q_{k}$ of $z^{k}$ as in (A.1)

$$
q_{k}= \begin{cases}-\theta \phi+\theta \sum_{j \geqslant 1}\left(\frac{-q}{1-q}\right)^{j}\left(\frac{\phi^{j}}{j}-\frac{\phi^{j+1}}{j+1}\right) & k=0, \\
\frac{\theta \phi^{k}}{(1-q)^{k}} \sum_{j \geqslant k}\left(\begin{array}{l}
j \\
k
\end{array}\right)\left(\frac{-q}{1-q}\right)^{j-k}\left(\frac{\phi^{j-k}}{j}-\frac{\phi^{j-k+1}}{j+1}\right) & k \geqslant 1 .\end{cases}
$$

For $q_{0}$ we have the series of a logarithm. For $k \geqslant 1$ we rewrite $q_{k}$ as two separate sums, and re-index the summations from zero

$q_{k}=\theta\left(\frac{\phi}{1-q}\right)^{k}\left[\frac{1}{k} \sum_{j \geqslant 0}\left(\begin{array}{c}k-1+j \\ k-1\end{array}\right) x^{j}+\phi \sum_{j \geqslant 0} \frac{(1+k)_{j}(1+k)_{j}}{(2+k)_{j}} \frac{x^{j}}{j !}\right]$

with $x=-q \phi /(1-q)$. The first sum is $(1-x)^{-k}$ and the second sum is a hypergeometric function, which can be simplified using (C.11), so finally we arrive at (18).

\section{Appendix B. Large $\gamma$-asymptotics of the resistance probability}

We show the large $\gamma$ approximations of the resistance probability $p_{0}$ as given in (27). For this we utilize the following asymptotics for the hypergeometric function as presented in $[30,15.12(\mathrm{iii})]$. Adapted to our notation they state that for fixed $a, b, c, z \in \mathbb{C}$

$$
F\left(\begin{array}{c}
a, b \\
c+\gamma
\end{array} ; z\right) \sim \frac{\Gamma(c+\gamma)}{\Gamma(c-b+\gamma)} \sum_{k \geqslant 0} r_{k}(z)(b)_{k} \gamma^{-k-b}
$$

for large $\gamma$, where $r_{s}(z)$ are the coefficients of the expansion of a specific function

$$
\sum_{k \geqslant 0} r_{k}(z) t^{k}=\left(\frac{\mathrm{e}^{t}-1}{t}\right)^{b-1} \mathrm{e}^{t(1-c)}\left(1-z+z \mathrm{e}^{-t}\right)^{-a} .
$$

We set for simplicity $z=\frac{q}{q-1}$ and apply (C.10) to the hypergeometric function in (27), then

$$
(1+\gamma) F\left(\begin{array}{c}
1, \gamma \\
2+\gamma
\end{array} ; q^{-1}\right)=(1+\gamma)(1-q) F\left(\begin{array}{c}
1,2 \\
2+\gamma
\end{array} ; z^{-1}\right)
$$

We can now apply (B.1)

$$
F\left(\begin{array}{c}
1,2 \\
2+\gamma
\end{array} ; z\right) \sim \frac{\gamma+1}{\gamma} \sum_{k \geqslant 0} r_{k}(z) \frac{(k+1) !}{\gamma^{k}}
$$


where $r_{k}(z)$ are the coefficient of the expansion of

$$
\frac{1-\mathrm{e}^{-t}}{t}\left(1-z+z \mathrm{e}^{-t}\right)^{-1}=1+(z-1 / 2) t+\left(z^{2}-z+1 / 6\right) t^{2}+O\left(t^{3}\right) .
$$

Using this expansion in (B.3) we arrive at (27). Note that the other similar results used throughout section 5.1 on the resistance probability can be proven in the same way and thus shall not be included here.

\section{Appendix C. Definitions and useful identities}

Euler's reflection formulas for the Gamma function are

$$
\begin{aligned}
& \Gamma(1+z) \Gamma(1-z)=\frac{\pi z}{\sin (\pi z)}, \\
& \Gamma(z+1) \Gamma(z-1)=\frac{z}{z-1} \Gamma(z)^{2} .
\end{aligned}
$$

The Digamma function is defined as

$$
\Psi(z)=\Psi_{0}(z)=\frac{\mathrm{d}}{\mathrm{d} z} \log \Gamma(z)=\frac{\Gamma^{\prime}(z)}{\Gamma(z)}
$$

and has the expansion around $z=0$

and around $z=\infty$

$$
\Psi(z)=-\frac{1}{z}-E_{C}+\frac{\pi^{2} z}{6}+O\left(z^{2}\right)
$$

$$
\Psi(z)=\log z-\frac{1}{2 z}-\frac{1}{12 z^{2}}+O\left(\frac{1}{z^{4}}\right)
$$

Its generalization is the Polygamma function

$$
\Psi_{n}(z)=\frac{\mathrm{d}^{n+1}}{\mathrm{~d} z^{n+1}} \log \Gamma(z) .
$$

The Pochhammer symbol is defined in terms of Gamma functions resp. as ascending factorial

$$
(a)_{b}=\frac{\Gamma(a+b)}{\Gamma(a)}=a(a+1)(a+2) \cdots(a+b-1) .
$$

The series of the hypergeometric function is

$$
F\left(\begin{array}{c}
a, b \\
c
\end{array} ; z\right)=\sum_{n \geqslant 0} \frac{(a)_{n}(b)_{n}}{(c)_{n}} \frac{z^{n}}{n !}
$$

for any (complex) $a, b, c$. The coefficients of the power series $\sum_{n \geqslant 0} A_{n} z^{n}$ of the hypergeometric function $F\left(\begin{array}{c}a, b \\ c\end{array} ;\right)$ satisfy $A_{0}=1$ and

$$
\frac{A_{n+1}}{A_{n}}=\frac{(n+a)(n+b)}{(n+c)(n+1)} \text {. }
$$

An alternative form of the hypergeometric function can be expressed as integral, if $c-b>0$

$$
F\left(\begin{array}{c}
a, b \\
c
\end{array} ; z\right)=\frac{\Gamma(c)}{\Gamma(b) \Gamma(c-b)} \int_{0}^{1} \frac{t^{b-1}(1-t)^{c-b-1}}{(1-z t)^{a}} \mathrm{~d} t .
$$


For $\Re(c-a-b)>0$ Gauß's hypergeometric Theorem states

$F\left(\begin{array}{c}a, b \\ c\end{array} 1\right)=\frac{\Gamma(c) \Gamma(c-a-b)}{\Gamma(c-a) \Gamma(c-b)}=\frac{(c-a-b)_{a}}{(c-a)_{a}}=\frac{(c-a-b)_{b}}{(c-b)_{b}}=\frac{(c-b)_{-a}}{(c)_{-a}}$,

which is also called the Chu-Vandermonde Identity if $a$ is a negative integer.

A useful identity

$$
F\left(\begin{array}{c}
a, b \\
c
\end{array} ; z\right)=(1-z)^{-b} F\left(\begin{array}{c}
c-a, b \\
c
\end{array} ; \frac{z}{z-1}\right),
$$

when applied twice becomes

$$
F\left(\begin{array}{c}
a, b \\
c
\end{array} ; z\right)=(1-z)^{c-a-b} F\left(\begin{array}{c}
c-a, c-b \\
c
\end{array} z\right) .
$$

Some inversion formulae (see [30])

$$
\begin{aligned}
F\left(\begin{array}{c}
a, b \\
c
\end{array} z\right)= & \frac{\Gamma(c) \Gamma(b-a)}{\Gamma(b) \Gamma(c-a)}(1-z)^{-a} F\left(\begin{array}{c}
a, c-b \\
a-b+1
\end{array} ; \frac{1}{1-z}\right) \\
& +\frac{\Gamma(c) \Gamma(a-b)}{\Gamma(a) \Gamma(c-b)}(1-z)^{-b} F\left(\begin{array}{c}
b, c-a \\
b-a+1
\end{array} ; \frac{1}{1-z}\right), \\
F\left(\begin{array}{c}
a, b \\
c
\end{array} z\right)= & \frac{\Gamma(c) \Gamma(c-a-b)}{\Gamma(c-b) \Gamma(c-a)} F\left(\begin{array}{c}
a, b \\
a+b-c+1
\end{array} ; 1-z\right) \\
& +\frac{\Gamma(c) \Gamma(a+b-c)}{\Gamma(a) \Gamma(b)} z^{1-c}(1-z)^{c-a-b} F\left(\begin{array}{c}
1-a, 1-b \\
c-a-b+1
\end{array} ; 1-z\right) .
\end{aligned}
$$

The derivative of the Hypergeometric function

$$
\frac{\mathrm{d}}{\mathrm{d} z} F\left(\begin{array}{c}
a, b \\
c
\end{array} ; z\right)=\frac{a b}{c} F\left(\begin{array}{c}
a+1, b+1 \\
c+1
\end{array} ; z\right) .
$$

Occasionally we need the limit behavior for $z \nearrow 1$. For general parameters the following formulas hold: If $c=a+b$, then

$$
\lim _{z \nearrow 1} \frac{F\left(\begin{array}{c}
a, b \\
a+b
\end{array} ; z\right)}{-\log (1-z)}=\frac{\Gamma(a+b)}{\Gamma(a) \Gamma(b)} .
$$

If $\Re(c-a-b)<0$, then

$$
\lim _{z \nearrow 1} \frac{F\left(\begin{array}{c}
a, b \\
a+b
\end{array} ; z\right)}{(1-z)^{c-a-b}}=\frac{\Gamma(c) \Gamma(a+b-c)}{\Gamma(a) \Gamma(b)} .
$$

Note that the Chu-Vandermonde identity is also the limit case for $\Re(c-a-b)>0$.

For a specific choice of the parameters the hypergeometric function can be expressed in simpler terms

$$
\begin{aligned}
& F\left(\begin{array}{c}
1,1 \\
2
\end{array} ; z\right)=-\frac{\log (1-z)}{z} \\
& F\left(\begin{array}{c}
1,1 \\
3
\end{array} ; z\right)=\frac{2(z+(1-z) \log (1-z))}{z^{2}}
\end{aligned}
$$

For general $z$ we can develop the hypergeometric function into

$$
F\left(\begin{array}{c}
1, b \\
c
\end{array} ; z\right)=1+\frac{b}{c} z F\left(\begin{array}{c}
1, b+1 \\
c+1
\end{array} ; z\right)
$$

Note that this formula is not valid for $a \neq 1$. 


\section{Appendix D. Theorems from singularity analysis}

Theorem VI.1 [29]: (Standard function scale). Let $a$ be an arbitrary complex number in $\mathbb{C} \backslash \mathbb{Z}_{\leqslant 0}$. The coefficient of $z^{n}$ for large $n$ has a full asymptotic expansion in descending powers of $n$

$$
\begin{aligned}
{\left[z^{n}\right](1-z)^{-a} } & \sim \frac{n^{a-1}}{\Gamma(a)}\left(1+\sum_{k \geqslant 1} \frac{e_{k}}{n^{k}}\right) \\
& \sim \frac{n^{a-1}}{\Gamma(a)}\left(1+\frac{a(a-1)}{2 n}+\frac{a(a-1)(a-2)(3 a-1)}{24 n^{2}}+\ldots\right)
\end{aligned}
$$

where $e_{k}$ is a polynomial in $a$ of degree $2 k$ and specified in [29].

Theorem VI.2 [29]: (Standard function scale, logarithms). Let $a$ be an arbitrary complex number in $\mathbb{C} \backslash \mathbb{Z}_{\leqslant 0}$ and $b \in \mathbb{C}$. The coefficient of $z^{n}$ for large $n$ has a full asymptotic expansion in descending powers of $n$

$$
\left[z^{n}\right](1-z)^{-a}\left(\frac{L}{z}\right)^{b} \sim \frac{n^{a-1}}{\Gamma(a)}(\log n)^{b}\left[1+\frac{C_{1}}{\log n}+\frac{C_{2}}{\log ^{2} n}+\ldots\right]
$$

where $C_{k}=\left.\left(\begin{array}{l}b \\ k\end{array}\right) \Gamma(a) \frac{\mathrm{d}^{k}}{\mathrm{~d} s^{k}} \frac{1}{\Gamma(s)}\right|_{s=a}$ and $L \equiv L(z)=\log \frac{1}{1-z}$. (Note that there is an erroneous $(-1)^{k}$ factor in the online version of the book.)

For $b=1$ we only have two terms

$$
\left[z^{n}\right](1-z)^{-a} \frac{L}{z} \sim \frac{n^{a-1}}{\Gamma(a)}[\log n-\Psi(a)]
$$

where $\Psi(a)=\Gamma^{\prime}(a) / \Gamma(a)$ is the Digamma function.

For $a \in \mathbb{Z}_{\leqslant 0}$ and $b \in \mathbb{Z}_{\geqslant 0}$

$$
\left[z^{n}\right](1-z)^{-a} L^{b} \sim n^{a-1}\left[F_{0}(\log n)+\frac{F_{1}(\log n)}{n}+\ldots\right],
$$

where the degree of $F_{n}$ is $k-1$. The polynomials are given by the following FrobeniusJungen method. For $b \in \mathbb{Z}_{\geqslant 0}$ and arbitrary $a \in \mathbb{C}$

$$
\left[z^{n}\right](1-z)^{-a} L^{b}=\frac{\mathrm{d}^{b}}{\mathrm{~d} a^{b}}\left(\begin{array}{c}
n+a-1 \\
n
\end{array}\right) .
$$

Note also that (D.5) is exact apart from finitely many terms. For example for $b=1$ and 2 , it takes the form

where

$$
\begin{aligned}
& {\left[z^{n}\right](1-z)^{-a} L=\left(\begin{array}{c}
n+a-1 \\
n
\end{array}\right) h_{n}(a)} \\
& {\left[z^{n}\right](1-z)^{-a} L^{2}=\left(\begin{array}{c}
n+a-1 \\
n
\end{array}\right)\left[h_{n}^{\prime}(a)-h_{n}^{2}(a)\right],}
\end{aligned}
$$

$$
\begin{aligned}
& h_{n}(a)=\Psi(a+n)+\Psi(n), \\
& h_{n}^{\prime}(a)=\Psi_{1}(a+n)+\Psi_{1}(n) .
\end{aligned}
$$

For negative integer $a$ consider the expression as a limit. For example for $b=1$ it simplifies to

$$
\left[z^{n}\right](1-z)^{k} L=(-1)^{k} \frac{k !}{n(n-1) \cdots(n-k)}
$$


and for $b=2$

$$
\left[z^{n}\right](1-z)^{k} L^{2}=2(-1)^{k} k ! \frac{C_{\mathrm{E}}+\Psi(n-k)-H_{k}}{n(n-1) \cdots(n-k)},
$$

where $H_{n}=\sum_{k=1}^{n} 1 / n$ is the $n$-th harmonic number.

\section{References}

[1] Luria S E and Delbrück M 1943 Mutations of bacteria from virus sensitivity to virus resistance Genetics 28 491-511

[2] Kessler D A and Levine H 2013 Large population solution of the stochastic Luria-Delbrück evolution model Proc. Natl Acad. Sci. USA $11011682-7$

[3] Lea D and Coulson C 1949 The distribution of the numbers of mutants in bacterial populations J. Genet. $49264-85$

[4] Bailey N T J 1964 The Elements of Stochastic Processes with Applications to the Natural Sciences (New York: Wiley)

[5] Zheng Q 1999 Progress of a half century in the study of the Luria-Delbrück distribution Math. Biosci. $1621-32$

[6] Kendall D G 1960 Birth-and-death processes, and the theory of carcinogenesis Biometrika 47 13-21

[7] Antal T and Krapivsky P L 2011 Exact solution of a two-type branching process: models of tumor progression J. Stat. Mech. P08018

[8] Clayton E, Doupe D P, Klein A M, Winton D J, Simons B D and Jones P H 2007 A single type of progenitor cell maintains normal epidermis Nature $446185-9$

[9] Antal T and Krapivsky P L 2010 Exact solution of a two-type branching process: clone size distribution in cell division kinetics J. Stat. Mech. P07028

[10] Bozic I et al 2013 Evolutionary dynamics of cancer in response to targeted combination therapy eLife 2 e00747

[11] Vogelstein B, Papadopoulos N, Velculescu V E, Zhou S, Diaz L A and Kinzler K W 2013 Cancer genome landscapes Science 339 1546-58

[12] Nowak M A 2006 Evolutionary Dynamics. Exploring the Equations of Life (Cambridge, MA: The Belknap Press of Harvard University Press)

[13] Angerer W P 2001 An explicit representation of the Luria-Delbrück distribution J. Math. Biol. 42 145-74

[14] Iwasa Y, Nowak M A and Michor F 2006 Evolution of resistance during clonal expansion Genetics $1722557-66$

[15] Komarova N L, Wu L and Baldi P 2007 The fixed-size Luria-Delbrück model with nonzero death rate Math. Biosci. 210 253-90

[16] Kessler D A and Levine H 2014 Scaling solution in the large population limit of the general asymmetric stochastic Luria-Delbrück evolution process J. Stat. Phys. doi:10.1007/s10955-014-1143-3

[17] Durrett R and Moseley S 2010 Evolution of resistance and progression to disease during clonal expansion of cancer Theor. Popul. Biol. 77 42-8

[18] Mandelbrot B 1974 A population birth-and-mutation process, I: explicit distributions for the number of mutants in an old culture of bacteria J. Appl. Probab. 11 437-44

[19] Möhle M 2005 Convergence results for compound poisson distributions and applications to the standard Luria-Delbrück distribution J. Appl. Probab. 42 620-31

[20] Janson S 2006 Limit theorems for triangular urn schemes Probab. Theory Relat. Fields 134 417-52

[21] Perry M C (ed) 2012 Perry's The Chemotherapy Source Book 5th edn (Philadelphia, PA: Lippincott Williams and Wilkins)

[22] Dewanji A, Luebeck E and Moolgavkar S 2005 A generalized Luria-Delbrück model Math. Biosci. 197 140-52

[23] Pakes A G 1993 Remarks on the Luria-Delbrück distribution J. Appl. Probab. 30 991-4

[24] Prodinger H 1996 Asymptotics of the Luria-Delbrück distribution via singularity analysis J. Appl. Probab. 33 282-3

[25] Arthreya K B and Ney P 2004 Branching Processes (New York: Dover)

[26] Ma W T, Sandri G V and Sarkar S 1992 Analysis of the Luria-Delbrück distribution using discrete convolution powers J. Appl. Probab. 29 255-67

[27] Kemp A W 1994 Comments on the Luria-Delbrück distribution J. Appl. Probab. 31 822-8

doi:10.1088/1742-5468/2015/01/P01011 
Mutant number distribution in an exponentially growing population

[28] Durrett R 1996 Probability: Theory and Examples (Cambridge Series in Statistical and Probabilistic Mathematics) (Cambridge: Cambridge University Press)

[29] Flajolet P and Sedgewick R 2009 Analytic Combinatorics (Cambridge: Cambridge University Press)

[30] NIST Digital Library of Mathematical Functions http://dlmf.nist.gov/, Release 1.0.7 of 2014-03-21. Online companion to [31]

[31] Olver F W J, Lozier D W, Boisvert R F and Clark C W 2010 NIST Handbook of Mathematical Functions (New York: Cambridge University Press). Print companion to [30] 